\title{
SIMPLE ENDOTRIVIAL MODULES FOR QUASI-SIMPLE GROUPS
}

\author{
CAROLINE LASSUEUR, GUNTER MALLE AND ELISABETH SCHULTE \\ Dedicated to Geoffrey Robinson on the occasion of his 60th birthday
}

\begin{abstract}
We investigate simple endotrivial modules of finite quasi-simple groups and classify them in several important cases. This is motivated by a recent result of Robinson 41] showing that simple endotrivial modules of most groups come from quasi-simple groups.
\end{abstract}

\section{INTRODUCTION}

Let $G$ be a finite group and $k$ a field of prime characteristic $p$ such that $p$ divides $|G|$. A $k G$-module $V$ is called endotrivial if $V \otimes V^{*} \cong k \oplus P$, with a projective $k G$-module $P$. The tensor product of $k G$-modules induces a group structure on the set of isomorphism classes of indecomposable endotrivial $k G$-modules, called the group of endotrivial modules and denoted $T(G)$. Endotrivial modules have seen a considerable interest in the last fifteen years, eventually leading to the determination of the Dade group for all $p$-groups (see, for example, [45] and the references therein), and of $T(G)$ for some classes of general groups (see [7, 8, 9, 10, 36]).

A recent paper of Robinson [41] has put the focus on simple endotrivial modules for quasi-simple groups. He shows that whenever the Sylow $p$-subgroups of a finite group $G$ are neither cyclic nor quaternion, then any simple endotrivial $k G$-module is either simple endotrivial for a quasi-simple normal subgroup, or induced from a 1-dimensional module of a strongly $p$-embedded subgroup of $G$. Note that, in contrast, for $p$-solvable groups of $p$-rank $>1$ all simple endotrivial modules are 1-dimensional by Navarro-Robinson [39].

The purpose of the present paper is to start a classification of simple endotrivial modules for quasi-simple groups. We obtain a complete description of such modules in several important cases. Our first main result is a precise condition for the existence of faithful simple endotrivial modules in the case of cyclic Sylow $p$-subgroups, depending on their location on the Brauer tree (see Theorem 3.7), and a complete classification for all such cases in quasi-simple groups not of classical Lie type or of rank at least 4, up to a small number of cases in sporadic groups in which the information on Brauer trees is yet incomplete.

Our second main result is the complete classification of simple endotrivial modules for covering groups of alternating groups, see Theorem 4.9.

Date: September 17, 2018.

2010 Mathematics Subject Classification. Primary 20C20; Secondary 20C30, 20C33, 20 C34.

Key words and phrases. simple endotrivial modules, cyclic defect, quasi-simple groups.

The authors gratefully acknowledge financial support by ERC Advanced Grant 291512. 
The third main result concerns groups of Lie type: for $p$ the defining characteristic we obtain a complete classification of all simple endotrivial modules in Theorem 5.2 , for nondefining characteristic we determine all simple endotrivial modules of exceptional groups of rank less than four and their covering groups.

Our results also indicate the validity of stronger statements: the existence of faithful simple endotrivial modules seems to drastically restrict the structure of Sylow $p$ subgroups:

Conjecture 1.1. Let $G$ be a finite quasi-simple group with a faithful simple endotrivial module. Then the Sylow p-subgroups of $G$ have rank at most 2.

Here, the rank of a $p$-group $H$ is the maximal rank of an elementary abelian subgroup of $H$. In fact, in all known examples, the Sylow $p$-subgroups are either homocyclic of rank at most 2 , extraspecial of order $p^{3}$ with $p \leq 11$, or dihedral (in $\mathrm{L}_{2}(q)$ with $q \equiv-1$ $(\bmod 4)$, see Proposition 3.8). As a consequence of our classifications we obtain:

Theorem 1.2. Conjecture 1.1 holds in all of the following cases:

(a) if $p=2$,

(b) if $G / Z(G)$ is an alternating group,

(c) if $G / Z(G)$ is a sporadic group,

(d) if $G / Z(G)$ is a group of Lie type and $p$ is its defining characteristic, and

(e) if $G / Z(G)$ is an exceptional group of Lie type.

Part (a) of this claim is shown in Theorem 6.7, part (b) follows from Theorem 4.9, part (c) from Theorem 7.1, part (d) from Theorem 5.2, and part (e) is Theorem 6.11. An a priori proof of Conjecture 1.1 would considerably simplify the classification of simple endotrivial modules.

The new feature of our approach is that the proofs rely mainly on character theoretic methods. This is made possible through our generalization of a lifting result due to Alperin [1] from the case of $p$-groups to arbitrary finite groups, which may be of independent interest:

Theorem 1.3. Let $(K, \mathcal{O}, k)$ be a p-modular system and $V$ be an endotrivial $k G$-module. Then $V$ lifts to an endotrivial $\mathcal{O} G$-module.

Previous work on endotrivial modules for different classes of quasi-simple groups $G$ was concerned with the determination of the group $T(G)$ of endotrivial modules. This includes results on groups with cyclic Sylow subgroups [36], the symmetric and alternating groups [8, 10], and groups of Lie type in their defining characteristic [9]. Nevertheless there are three main obstructions to use these results to answer the current question of finding the simple endotrivial modules. First, the aforementioned articles do not treat covering groups. Second, they determine the structure of $T(G)$ but not the indecomposable endotrivial modules themselves, in that their description involves Green correspondence, which is not explicit and notoriously difficult to determine. Third, even in the simplest cases where $T(G)=\langle\Omega(k)\rangle \cong \mathbb{Z}$ (where $\Omega$ is the Heller operator), it is not clear whether any of the modules $\Omega^{n}(k)$ for $n \in \mathbb{Z}$ can be simple. Finally, even in those few cases where the cited papers obtain explicit descriptions of some simple endotrivial modules, our approach seems more straightforward than the more intricate module theoretic methods. 
Finally, a comparison of our results with those of [7, 8, 9, 10, 36] seems to indicate a strong link between the order of the torsion subgroup of $T(G)$ and the number of simple endotrivial modules. Therefore a classification of simple endotrivial modules may appear to be an important step towards the final description of the group $T(G)$. Our study of sporadic groups via character-theoretic methods also provides us with new torsion elements of $T(G)$ unknown in the literature so far, see Remark 7.2 .

The paper is built up as follows: in Section 2 we collect some basic facts and prove Theorem [1.3. In Section 3 we obtain rather strong results in the case of cyclic Sylow subgroups. In Section 4 we classify the simple endotrivial modules for covering groups of alternating groups, and in Section 5 those for groups of Lie type in their defining characteristic. In Section 6 we obtain partial results for simple groups of Lie type, mostly of exceptional type, in cross characteristic, and in the final section we classify simple endotrivial modules for sporadic groups and their covering groups, up to a few open cases of very large dimension.

Acknowledgement: We thank the anonymous referee for her/his numerous detailed remarks and comments which led to an improvement of our paper.

\section{Preliminaries}

Throughout, unless otherwise stated, we assume $G$ is a finite group and $k$ an algebraically closed field of prime characteristic $p$ such that $p$ divides $|G|$. We let $(K, \mathcal{O}, k)$ be a splitting $p$-modular system, and let $\mathfrak{p}:=J(\mathcal{O})$. For a block $\mathbf{B}$ of $k G$ we write $\operatorname{se}(\mathbf{B})$ for the number of isomorphism classes of simple endotrivial B-modules. For background material on endotrivial modules we refer to $[7,8,9,10,36]$. We first collect some elementary facts.

Lemma 2.1. Let $V$ be an endotrivial $k G$-module, with $k$ a field of characteristic $p$. Then $\operatorname{dim} V \equiv \pm 1\left(\bmod |G|_{p}\right)$ for $p \geq 3$, respectively $\operatorname{dim} V \equiv \pm 1\left(\bmod \frac{1}{2}|G|_{2}\right)$ when $p=2$.

Proof. Since $V$ is endotrivial, $V \otimes V^{*} \cong k \oplus$ (projective), so $(\operatorname{dim} V)^{2}-1 \equiv 0\left(\bmod |G|_{p}\right)$. When $p>2$, this means that either $\operatorname{dim} V+1$ or $\operatorname{dim} V-1$ is divisible by $|G|_{p}$, while for $p=2$, at least one of the factors is divisible by $\frac{1}{2}|G|_{2}$.

Lemma 2.2. Let $V$ be a $k G$-module and $H \leq G$ containing a Sylow p-subgroup of $G$. Then $V$ is endotrivial if and only if $\left.V\right|_{H}$ is endotrivial.

Proof. The claim follows immediately from the fact that a $k G$-module is projective if and only if its restriction to a Sylow $p$-subgroup of $G$ is projective.

Corollary 2.3. Let $V$ be a $k G$-module which is liftable to a simple $\mathbb{C} G$-module with character $\chi$, say. If $V$ is endotrivial, then $|\chi(g)|=1$ for all p-singular elements $g \in G$.

Proof. By assumption $\chi \bar{\chi} \equiv 1+\psi(\bmod p)$, where $\psi$ is the character of a $p$-projective module. Thus, $\psi(g)=0$ for all $p$-singular elements $g \in G$. The claim follows.

We now prove Theorem 1.3 from the introduction. This result is due to Alperin for $p$ groups [1] (for which the image of any representation lies in the special linear group). His proof generalises easily. We sum up here the main ideas, based on a detailed exposition of the proof for $p$-groups written in [47]. 
Proposition 2.4. Let $V$ be an endotrivial $k G$-module such that the image of the corresponding representation $\rho: G \rightarrow \mathrm{GL}_{n}(k)$ lies in $\mathrm{SL}_{n}(k)$. Then $V$ lifts to an endotrivial $\mathcal{O} G$-module.

Proof. Denote by $\mathrm{SL}_{n}(\mathcal{O}, m)$ the congruence subgroups of $\mathrm{SL}_{n}(\mathcal{O})$, i.e., the set of determinant 1 matrices congruent to the identity matrix $I_{n}$ modulo $\mathfrak{p}^{m}$. They form a central series of $\mathrm{SL}_{n}(\mathcal{O})$ with successive quotients all isomorphic to $\mathfrak{s l}_{n}(k)$ (the $n \times n$-matrices of trace zero) as $\mathrm{SL}_{n}(k)$-modules. Since $V$ is endotrivial, $\operatorname{End}_{k}(V) \cong k \oplus$ (projective), and $n \equiv \pm 1(\bmod p)$ by Lemma 2.1 , thus we also have $\operatorname{End}_{k}(V) \cong k \oplus U$ where $U$ is the kernel of the trace map on $\operatorname{End}_{k}(V)$. Hence $\mathfrak{s l}_{n}(k)$, seen as a $k G$-module via $\rho$, must be projective.

Taking a pull-back $X_{2}$ of $\rho$ and the homomorphism induced by reduction modulo $\mathfrak{p}$ from $\mathrm{SL}_{n}(\mathcal{O}) / \mathrm{SL}_{n}(\mathcal{O}, 2) \rightarrow \mathrm{SL}_{n}(k)$, which has kernel $\mathrm{SL}_{n}(\mathcal{O}, 1) / \mathrm{SL}_{n}(\mathcal{O}, 2) \cong \mathfrak{s l}_{n}(k)$, yields a group extension $1 \rightarrow \mathfrak{s l}_{n}(k) \rightarrow X_{2} \rightarrow G \rightarrow 1$. This extension splits because $\mathfrak{s l}_{n}(k)$ is a projective (= injective) $k G$-module. As a consequence, $\rho$ lifts to a homomorphism $\rho_{2}: G \rightarrow \mathrm{SL}_{n}(\mathcal{O}) / \mathrm{SL}_{n}(\mathcal{O}, 2)$. Inductively, for every $m>2$, one thus constructs a homomorphism $\rho_{m}: G \rightarrow \mathrm{SL}_{n}(\mathcal{O}) / \mathrm{SL}_{n}(\mathcal{O}, m)$ lifting $\rho_{m-1}: G \rightarrow \mathrm{SL}_{n}(\mathcal{O}) / \mathrm{SL}_{n}(\mathcal{O}, m-1)$. Finally $\mathrm{SL}_{n}(\mathcal{O}) \cong \lim _{m \geq 2} \mathrm{SL}_{n}(\mathcal{O}) / \mathrm{SL}_{n}(\mathcal{O}, m)$, so that the universal property of the projective limit yields the desired group homomorphism $\tilde{\rho}: G \longrightarrow \mathrm{SL}_{n}(\mathcal{O})$ lifting $\rho$.

Moreover if $M$ is an $\mathcal{O} G$-module lifting $V$, then $\operatorname{rk}_{\mathcal{O}}(M)=\operatorname{dim}_{k}(V) \equiv \pm 1(\bmod p)$ by Lemma 2.1, so that $\operatorname{End}_{\mathcal{O}}(M) \cong \mathcal{O} \oplus N$ with $N$ the kernel of the trace map on $\operatorname{End}_{\mathcal{O}}(M)$. Reducing modulo $\mathfrak{p}$ yields $\operatorname{End}_{k}(V) \cong k \oplus N / \mathfrak{p} N$, where $N / \mathfrak{p} N$ is projective by assumption. It follows that $N$ is projective, see e.g. [44, $\S 1$ and $\S 27$ ]. Hence $M$ is endotrivial.

Proof of Theorem 1.3. By passing to the image of the representation and choosing a basis of $V$ we may assume that $G \leq \mathrm{GL}_{n}(k)$. Let $G_{1}:=G C$ and $G_{0}:=G_{1} \cap \mathrm{SL}_{n}(k)$, with

$$
C:=\left\{a I_{n} \mid a^{n}=\operatorname{det}(g) \text { for some } g \in G\right\} \text {. }
$$

Then $G_{1}$ is a central product of $G$ with $C$, and of $G_{0}$ and $C$. As $\left|G_{1}: G\right|$ and $\left|G_{1}: G_{0}\right|$ are prime to $p$, the embedding $G_{0} \leq \mathrm{SL}_{n}(k)$ is also endotrivial. Thus by Proposition 2.4 it lifts to an endotrivial $\mathcal{O} G_{0}$-module. Denoting the corresponding representation by $\psi$ we thus have $\psi\left(G_{0}\right) \leq \operatorname{SL}_{n}(\mathcal{O})$.

Reduction modulo $\mathfrak{p}$ induces a bijection between the group of $p^{\prime}$-roots of unity in $\mathcal{O}$ and roots of unity in $k$, sending $\psi\left(G_{0}\right) \cap Z\left(\mathrm{SL}_{n}(\mathcal{O})\right)$ onto $G_{0} \cap Z\left(\mathrm{SL}_{n}(k)\right)$. The inverse defines a lift of $C$ into $\left\{a I_{n} \mid a \in \mathcal{O}^{\times}\right\} \leq \mathrm{GL}_{n}(\mathcal{O})$, which agrees with $\psi$ on $G_{0} \cap Z\left(\mathrm{SL}_{n}(k)\right)$ and which we also denote by $\psi$. Then $G_{1}=G_{0} C \cong \psi\left(G_{0}\right) \psi(C) \leq \mathrm{GL}_{n}(\mathcal{O})$ is a faithful representation of $G_{1}$ which lifts $G_{1} \leq \mathrm{GL}_{n}(k)$. Again, as $\left|G_{1}: G\right|$ and $\left|G_{1}: G_{0}\right|$ are prime to $p$, this gives an endotrivial $\mathcal{O} G$-module lifting the initial representation of $G$.

\section{Groups with CyCliC Sylow p-Subgroups}

In this section $k$ denotes an algebraically closed field of characteristic $p>0$, and $G$ denotes a finite group with a non-trivial cyclic Sylow $p$-subgroup $P \cong C_{p^{n}}$ for some integer $n \geq 1$.

For any group $G$, denote by $T(G)$ its group of endotrivial modules and by $X(G)$ the subgroup of $T(G)$ consisting of the one-dimensional $k G$-modules (with group law induced 
by $\left.\otimes_{k}\right)$. This group can be identified with the group of $k^{\times}$-valued linear characters of $G$ so that $X(G) \cong(G /[G, G])_{p^{\prime}}$, the $p^{\prime}$-part of the abelianization of $G$.

Let $Z$ denote the unique subgroup of $P$ of order $p$ and let $H:=N_{G}(Z)$. The structure of the group $T(G)$ is described in [36] as follows: since $H$ is strongly p-embedded in $G, T(G) \cong T(H)$ via restriction and inverse map induced by Green correspondence. Furthermore, there is an exact sequence

$$
0 \longrightarrow X(H) \longrightarrow T(H) \stackrel{\operatorname{Res}_{P}^{H}}{\longrightarrow} T(P) \longrightarrow 0
$$

so that

$$
T(H)= \begin{cases}X(H) & \text { if }|P|=2 ; \\ \left\langle X(H),\left[\Omega\left(k_{H}\right)\right]\right\rangle & \text { if }|P| \geq 3 .\end{cases}
$$

In addition, $T(P)=\left\langle\left[\Omega\left(k_{P}\right)\right]\right\rangle \cong \mathbb{Z} / 2$ if $|P| \geq 3$ and $T(P)=\{0\}$ if $|P|=2$ by [14]. Unless otherwise stated, we assume for the rest of the section that $|P| \geq 3$, in which case $|T(H): X(H)|=2$.

3.1. Endotrivial modules and blocks of $k G$. Indecomposable endotrivial modules have dimension prime to $p$, thus have the Sylow subgroups as vertices and lie in blocks with full defect. Henceforth $\mathbf{B}$ denotes a block of $k G$ with defect group $P$, and $e_{\mathbf{B}}$ denotes its inertial index, which corresponds to the number of simple modules in B. Moreover let $\mathbf{B}_{0}$ denote the principal block of $k G$ and set $e:=e_{\mathbf{B}_{0}}=\left|N_{G}(Z): C_{G}(Z)\right|$, so that $e \mid p-1$.

If $M$ is an indecomposable non-projective $k G$-module, denote by $f(M)$ its $k H$-Green correspondent. If $L$ is an indecomposable non-projective $k H$-module, denote by $g(L)$ its $k G$-Green correspondent. If $M$ belongs to $\mathbf{B}$, then $f(M)$ belongs to the Brauer correspondent $\mathbf{b}$ of $\mathbf{B}$.

The stable Auslander-Reiten quiver $\Gamma_{s}(\mathbf{B})$ of $\mathbf{B}$ is a finite tube $\left(\mathbb{Z} / e_{\mathbf{B}} \mathbb{Z}\right) A_{p^{n}-1}$, so that any non-projective indecomposable $k G$-module has $\Omega$-period $2 e_{\mathbf{B}}$. For background material and standard notation and terminology on the Auslander-Reiten quiver, we refer the reader to [2, Chap. $4 \&$ Sec. 6.5]. We recall that Green correspondence sets up an $\Omega^{2}$ equivariant graph isomorphism between $\Gamma_{s}(\mathbf{B})$ and $\Gamma_{s}(\mathbf{b})$. Furthermore the structure of the $k H$-Brauer correspondent $\mathbf{b}$ of a block $\mathbf{B}$ of $k G$ is well-known: $\mathbf{b}$ has $e_{\mathbf{b}}=e_{\mathbf{B}}$ simple modules, all of the same $k$-dimension. These $e_{\mathbf{b}}$ simple modules form one boundary $\Omega^{2}$-orbit of $\Gamma_{s}(\mathbf{b})[2$, Sec. 6.5]. Nonetheless, the $k G$-Green correspondent of a simple $k H$ module is not necessarily simple. Simple $\mathbf{B}$-modules lie in the $e$ top and bottom $\Omega^{2}$-orbits of $\Gamma_{s}(\mathbf{B})$, each of them on a different diagonal [3, Prop. 4.2].

Lemma 3.1. Let $\mathbf{B}$ be a block of $k G$ containing an indecomposable endotrivial module $V$. Then:

(a) $e_{\mathbf{B}}=e$.

(b) $\mathbf{B}$ contains $2 e$ endotrivial modules. They are exactly the modules forming the two boundary $\Omega^{2}$-orbits of $\Gamma_{s}(\mathbf{B})$.

(c) The $\Omega^{2}$-orbit of $\Gamma_{s}(\mathbf{B})$ containing $V$ consists of the modules $\Omega^{2 n}(V), 1 \leq n \leq e$, while the other boundary $\Omega^{2}$-orbit consists of the modules $\Omega^{2 n-1}(V), 1 \leq n \leq e$.

Proof. The connected component $A R(V)$ of $V$ in the stable Auslander-Reiten quiver of $k G$ is $\Gamma_{s}(\mathbf{B})$, a finite tube $\left(\mathbb{Z} / e_{\mathbf{B}} \mathbb{Z}\right) A_{p^{n}-1}$. Now by [4, Thm. 2.3], $A R(V) \cong A R(k)=\Gamma_{s}\left(\mathbf{B}_{0}\right)$ which is a tube $(\mathbb{Z} / e \mathbb{Z}) A_{p^{n}-1}$. Hence $e_{\mathbf{B}}=e$. By [4, Thm. 2.6], the endotrivial modules 
in $\Gamma_{s}(\mathbf{B})$ are exactly the modules lying on the two boundary $\Omega^{2}$-orbits. Whence there are $2 e$ of them. Part (c) is well-known (see e.g. [3, §4]).

Lemma 3.2. Let $\mathbf{B}$ be a block of $k G$. Then:

(a) $\mathbf{B}$ contains an indecomposable endotrivial module if and only if its Brauer correspondent $\mathbf{b}$ contains a one-dimensional $k H$-module.

(b) If $V$ is an indecomposable endotrivial $\mathbf{B}$-module, then there exist $U \in X(H)$ such that either $V \cong g(U)$ or $V \cong g(\Omega(U))$.

(c) There are $|X(H)| /$ e blocks of $k G$ containing indecomposable endotrivial modules. Each of them contains at least one simple endotrivial module.

Proof. By [36, Thm. 3.6], $T(G) \cong T(H)$ via Green correspondence on the indecomposable endotrivial modules, i.e., an indecomposable B-module $V$ is endotrivial if and only if $f(V)$, lying in $\mathbf{b}$, is endotrivial. Now, the simple modules in $\mathbf{b}$ are all of the same dimension and form one boundary $\Omega^{2}$-orbit of $\Gamma_{s}(\mathbf{b})$. Thus parts (b) and (c) of Lemma 3.1 together with the fact that $|T(H): X(H)|=2$, imply that $T(H)$ contains exactly $|T(H)| / 2$ simple modules, all of dimension one. Whence (a). Part (b) then follows from parts (b) and (c) of Lemma 3.1. Finally again since $|T(G)|=|T(H)|=2|X(H)|$, parts (a) and (b) of Lemma 3.1 force the number of blocks containing endotrivial modules to be $|X(H)| / e$. Moreover each of them contains a simple endotrivial module by [3, Thm. 3.7], which proves that there is at least one simple $\mathbf{B}$-module lying on an end $\Omega^{2}$-orbit of $\Gamma_{s}(\mathbf{B})$.

Corollary 3.3. The number of simple endotrivial modules over $k G$ is bounded below by $|X(H)| / e$ and bounded above by $|X(H)|=|H /[H, H]|_{p^{\prime}}$.

Proof. The lower bound is given by Lemma 3.2(c). The upper bound follows by Lemma 3.1 from the fact that $|T(H): X(H)|=2$.

3.2. Location of simple endotrivial modules on the Brauer tree. Let $\mathbf{B}$ be a block of $k G$ and let $\sigma(\mathbf{B})$ denote its Brauer tree. Using notation of [24], the nodes of $\sigma(\mathbf{B})$ can be labelled by noughts $\circ$ and crosses $\times$, such that a nought can only be joined to a cross and a cross to a nought. Moreover, let $\chi_{0}$ denote the exceptional node of $\sigma(\mathbf{B})$, if it has exceptional multiplicity $m_{\mathbf{B}}:=\left(p^{n}-1\right) / e_{\mathbf{B}}>1$ and otherwise let $\chi_{0}$ be any node of $\sigma(\mathbf{B})$. If $S$ is a simple $\mathbf{B}$-module labelling an edge of $\sigma(\mathbf{B})$, let $n(S)$ be the number of nodes of $\sigma(\mathbf{B})$ which are not connected to $\chi_{0}$ after removal of the edge $S$. Let $l(f(S))$ denote the length of the $k H$-Green correspondent of $S$. Then the following holds:

Lemma 3.4. Let $S$ be a simple $\mathbf{B}$-module labelling an edge of $\sigma(\mathbf{B})$ and let $\chi$ be the node adjacent to $S$, which is not connected to $\chi_{0}$ after removal of the edge $S$. Then

$$
l(f(S))= \begin{cases}n(S) & \text { if } \chi \text { has type } \times, \\ p^{n}-n(S) & \text { if } \chi \text { has type } \circ .\end{cases}
$$

Proof. See [24, Lem. 4.4.11] and [16, Lem. 9.3].

If $S$ is a simple B-module, let $\times(S)$ denote the number of nodes on the part of $\sigma(\mathbf{B})$ to which the node adjacent to $S$ of type $\times$ belongs after removal of the edge $S$, with the exceptional node counted $\left(p^{n}-e\right)$-times.

Lemma 3.5. Let $S$ be a simple B-module. Then $l(f(S))=\times(S)$. 
Proof. [24, Lem. 4.4.12] states and proves the case $n=1$. Their proof can be generalized to an arbitrary $n$ : Let $S$ be an edge of $\mathbf{B}$ and let $\chi$ be the node adjacent to $S$, which is not connected to $\chi_{0}$ after removal of $S$. If $\chi$ is of type $\times$, then $\times(S)=n(S)=l(f(S))$ by Lemma 3.4. If $\chi$ is of type $\circ$, then by Lemma 3.4 we get

$$
l(f(S))=p^{n}-n(S)=p^{n}-\left(e+1-\left(\times(S)-\left(p^{n}-e\right)+1\right)\right)=\times(S) .
$$

Lemma 3.6. Let $\mathbf{B}$ be a block of $k G$ containing an endotrivial module and let $S$ be a simple B-module. Then $S$ is endotrivial if and only if $l(f(S)) \in\left\{1, p^{n}-1\right\}$.

Proof. Let $\mathbf{b}$ be the Brauer correspondent of $\mathbf{B}$. Then the claim follows from the fact that the boundary $\Omega^{2}$-orbits of $\Gamma_{s}(\mathbf{b})$ are made of the indecomposable $k H$-modules of length 1 and $p^{n}-1$, together with Lemma 3.1 and 3.2 .

Let us call a leaf of a Brauer tree $\sigma(\mathbf{B})$ an exceptional leaf if the exceptional node is sitting at the end of this leaf and has exceptional multiplicity $m_{\mathbf{B}}>1$. Then we can state the main result of this section.

Theorem 3.7. Let $\mathbf{B}$ be a block of $k G$ containing an endotrivial module and assume $e>1$. Let $S$ be a simple $\mathbf{B}$-module. Then $S$ is endotrivial if and only if $S$ corresponds to a non-exceptional leaf of $\sigma(\mathbf{B})$.

Proof. By definition the leafs of $\sigma(\mathbf{B})$ correspond to liftable simple B-modules whereas the inner edges correspond to non liftable simple modules. Hence by Theorem 1.3 only leaves of $\sigma(\mathbf{B})$ can be endotrivial. So let $S$ be a simple B-module corresponding to a leaf of $\sigma(\mathbf{B})$ and let $\chi$ denote the end node of this leaf. Then by Lemma 3.6, $S$ is endotrivial if and only if $l(f(S)) \in\left\{1, p^{n}-1\right\}$. Applying Lemma 3.5, we obtain that the length $l(f(S))$ is as follows:

If $\chi \neq \chi_{0}$ and is of type $\times$, then $l(f(S))=1$.

If $\chi \neq \chi_{0}$ and is of type $\circ$, then $l(f(S))=(e-1)+p^{n}-e=p^{n}-1$.

Notice that if $\chi_{0}$ has multiplicity one, then we may always assume that $\chi \neq \chi_{0}$. Therefore, now assume that $\chi_{0}$ has multiplicity $m_{\mathbf{B}}>1$, so $1<e<p-1$.

If $\chi=\chi_{0}$ and $\chi$ is of type $\times$, then $l(f(S))=p^{n}-e \notin\left\{1, p^{n}-1\right\}$.

If $\chi=\chi_{0}$ and $\chi$ is of type $\circ$, then $l(f(S))=e \notin\left\{1, p^{n}-1\right\}$. Hence the result.

3.3. $\mathrm{SL}_{2}(q)$ in cross characteristic. As an application we classify simple endotrivial modules for $G=\mathrm{SL}_{2}(q), q=p^{n}, p$ a prime, in non-defining characteristic $\ell$ dividing the order of $G$. For the exceptional covering groups of $\mathrm{L}_{2}(9) \cong \mathfrak{A}_{6}$ see Theorem 4.9 , For $\ell=p$, see Theorem 5.2.

Proposition 3.8. Let $G=\mathrm{SL}_{2}(q), q=p^{n}$ with $p$ a prime. Let $V$ be a non-trivial simple $k G$-module, where $k$ is algebraically closed of characteristic $\ell \neq p$. Then $V$ is endotrivial if and only if one of:

(1) $2 \neq \ell \mid q-1$ and $V$ lies in an $\ell$-block of full defect and inertial index 2 (cyclic defect);

(2) $p \neq 2 \neq \ell \mid q+1$ and $V$ lies in the non-principal $\ell$-block of full defect and inertial index 2 (cyclic defect);

(3) $3=\left.\ell|q+1| G\right|_{,\ell}=3$ and $V$ lies in the principal $\ell$-block (cyclic defect). 
Moreover, if $\ell=2, q \equiv-1(\bmod 4)$ and $V$ lies in the principal $\ell$-block, then $V$ is endotrivial as a $k \mathrm{~L}_{2}(q)$-module, but not as a $k G$-module.

This proposition is essentially proven in [42 via character theory. Only the cases $p \neq \ell=2, q \equiv-1(\bmod 4)$ and $p \neq 2 \neq \ell \mid q+1$ for the non-principal $\ell$-block of full defect and inertial index 2 were left open. We give here a summary proof using the techniques of this section and some results of Craven's [13].

Proof. Only blocks with full defect need to be investigated, for indecomposable endotrivial modules have Sylow subgroups as vertices. In addition, in the cyclic defect case, only blocks $\mathbf{B}$ with $e_{\mathbf{B}}=e$ can contain endotrivial modules by Lemma 3.1. Let $\mathbf{B}_{0}$ denote the principal $\ell$-block of $k G$. Recall that $\left|\mathrm{SL}_{2}(q)\right|=(q-1) q(q+1)$.

If $\ell \neq 2$ and $\ell \mid q-1$ or $\ell \mid q+1$, then a Sylow $\ell$-subgroup of $G$ is cyclic and $e=2$. Moreover if $p \neq 2$, then $G$ has exactly two $\ell$-blocks of full defect and inertial index $2, \mathbf{B}_{0}$ and say $\mathbf{B}_{1}$. By [5, $\S 6.2 .1$ and $\left.\S 6.2 .2\right],|X(H)|=4$, thus the number of blocks containing endotrivial modules is $|X(H)| / e=2$ by Lemma 3.2. Now if $\ell \mid q-1$, the Brauer trees $\sigma\left(\mathbf{B}_{0}\right), \sigma\left(\mathbf{B}_{1}\right)$ both have the form $\circ \_-\bullet$ with exceptional node in the middle. (See [5, Sec. 9.3 and §9.4.2]). Therefore, by Theorem 3.7, all non-trivial simple $\mathbf{B}_{0^{-}}$and $\mathbf{B}_{1^{-}}$ modules are endotrivial. If $\ell \mid q+1, \sigma\left(\mathbf{B}_{1}\right)$ has the form $\circ-\bullet-\circ$ with exceptional node in the middle (see [5, Sec. 9.3]), hence both simple $\mathbf{B}_{1}$-modules are endotrivial by Theorem 3.7. The tree $\sigma\left(\mathbf{B}_{0}\right)$ has the form $\circ-\circ-\bullet$ with exceptional node sitting on one end (see [5, §9.4.3]). Moreover the exceptional multiplicity is $m_{\mathbf{B}_{0}}=1$ if and only if $\ell=3$ and $|G|_{\ell}=3$. Thus, by Theorem 3.7, $\mathbf{B}_{0}$ contains a non-trivial simple endotrivial module if and only if $|G|_{\ell}=3$. (The trivial module corresponds to the non-exceptional end node.) Now if $p=2$, the situation is similar, except that only the principal block has full defect and inertial index 2 (see [5, §9.4.2]).

If $p \neq \ell=2$, then only the principal block has full defect: it contains two non-trivial simple modules $S_{1}$ and $S_{2}$ with $\operatorname{dim}\left(S_{1}\right)=\operatorname{dim}\left(S_{2}\right)=\frac{1}{2}(q-1)$ (see [5, Chap. 9]). If $q \equiv 1$ $(\bmod 4)$, then $\operatorname{dim}\left(S_{i}\right) \equiv 0(\bmod 2)$. Hence $S_{1}, S_{2}$ are not endotrivial by Lemma 2.1. If $q \equiv-1(\bmod 4)$, then $S_{1}$ and $S_{2}$ are endotrivial $k L_{2}(q)$-modules by [13, Sec. 4.4] for $q \equiv 3(\bmod 8)$ and by $[13$, Prop. 4.26$]$ for $q \equiv 7(\bmod 8)$. However, the inflation of $S_{1}$ and $S_{2}$ from $\mathrm{L}_{2}(q)=G / Z(G)$ to $G$ does not yield endotrivial $k G$-modules. Indeed, since $|Z(G)|=2$, we get for $1 \leq i \leq 2, \operatorname{End}_{k}\left(S_{i}\right) \cong k \oplus\left(\operatorname{Ind}_{Z(G)}^{G}(k) \oplus \cdots \oplus \operatorname{Ind}_{Z(G)}^{G}(k)\right)$ as $k G$-modules.

\section{Covering groups of Alternating Groups}

In this section we classify simple endotrivial modules for covering groups of alternating groups. Note that some (but not all) simple endotrivial modules for alternating groups were described in [8, 10, using different methods. The faithful modules for proper covering groups were not investigated previously.

Throughout $p$ denotes a prime and $k$ a large enough field of characteristic $p$.

4.1. Faithful modules for $\mathfrak{A}_{n}$. Recall that the simple $\mathbb{Q S}_{n}$-modules are parametrized by partitions of $n$. We first classify partitions possessing certain types of hooks.

Lemma 4.1. Let $n=m p+r$ with $0 \leq r<p$ and assume that $n \geq 2 p$. Let $\lambda \vdash n$ be a partition such that the corresponding irreducible character $\chi_{\lambda}$ of $\mathfrak{S}_{n}$ does not vanish on 
elements of cycle shape $(n-p)(p),(n-p-1)(p)(1)$, nor on elements whose cycle shape contains a cycle of length $n-r$. Then $\lambda$ or its conjugate is one of the partitions

$$
\left(1^{n}\right) \quad \text { or } \quad\left(n-p, r+1,1^{p-r-1}\right) .
$$

Proof. By the Murnaghan-Nakayama formula (see e.g. [29, 2.4.7]), if $\chi_{\lambda}$ does not vanish on elements whose cycle shape contains an $n-r$-cycle, then $\lambda$ must have a hook of length $n-r$, and the partition $\mu$ obtained by removing such a hook must have hooks of any length less or equal to $r$. Thus, by assumption $\mu$ is, up to conjugate, the partition $(r)$, and then $\lambda$ is of the form

$$
\left(r, b, 1^{n-r-b}\right) \quad(1 \leq b \leq r) \quad \text { or } \quad\left(r+a, r+1,1^{n-2 r-a-1}\right) \quad(1 \leq a \leq n-2 r-1),
$$

or $\lambda$ is the hook $\left(r, 1^{n-r}\right)$.

If $\chi_{\lambda}$ also vanishes on elements of cycle shape $(n-p)(p)$, then $\lambda$ has to possess a hook of length $n-p$, and the partition obtained by removing such a hook must be a hook itself (of length $p$ ). It is easily seen that for the above possibilities, if $\lambda=\left(r, b, 1^{n-r-b}\right)$ then we have $r=p-1$, so $\lambda=\left(p-1,1^{n-p+1}\right)$, if $\lambda=\left(r+a, r+1,1^{n-2 r-a-1}\right)$ then only the two partitions $\left(n-p, r+1,1^{p-r-1}\right)$ and $\left(p, r+1,1^{n-p-r-1}\right)$ are possible, and the only hook satisfying our condition is $\left(r, 1^{n-r}\right)$.

If finally $\chi_{\lambda}$ does not vanish on elements of cycle shape $(n-p-1)(p)$, then $\lambda$ has an $n-p-1$-hook, and the remaining partition has a $p$-hook. In our first case, this is seen not to be possible. In the second case, it holds true when $\lambda=\left(n-p, r+1,1^{p-r-1}\right)$, and finally, the hook $\left(r, 1^{n-r}\right)$ only has this property when $r=1$, so $\lambda=\left(1^{n}\right)$. The claim is shown.

Proposition 4.2. Any simple faithful endotrivial $k \mathfrak{A}_{n}$-module, $n \geq 2 p$, over a field $k$ of characteristic $p>2$ is a constituent of the restriction to $\mathfrak{A}_{n}$ of a $\mathbb{Q S}_{n}$-module indexed by $\lambda \vdash n$, where one of:

(1) $2 p \leq n=2 p+r \leq 3 p-1$ and $\lambda=\left(p+r, r+1,1^{p-r-1}\right)$;

(2) $n=2 p, \lambda=\left(p, 2,1^{p-2}\right)$; or

(3) $n=2 p+1, \lambda=\left(p+1,1^{p}\right)$.

Proof. By Clifford-theory any simple faithful $\mathcal{O A}_{n}$-module occurs in the restriction of some simple faithful $\mathcal{O S}_{n}$-module $V$. We distinguish two cases. First assume that $V$ restricts irreducibly. Then according to Lemma 2.2 it suffices to show that $V$ is not endotrivial for $\mathfrak{S}_{n}$ to conclude the same for $\mathfrak{A}_{n}$. For this we will show that any non-linear character $\chi_{\lambda}$ of $\mathfrak{S}_{n}$ except for those listed in (1) of the claim vanishes on some $p$-singular element, from which the assertion will follow by Corollary 2.3 .

Note that permutations of cycle shape $(n-p)(p),(n-p-1)(p)$ and $(n-r)(r)$ are $p$-singular, where $n=m p+r, 0 \leq r<p$. Thus, our claim already holds by Lemma 4.1 unless $\lambda=\left(n-p, r+1,1^{p-r-1}\right)$ or the conjugate partition. But $\lambda$ does not have a hook of length $n-r-p$ if $n \geq 3 p$, so $\chi_{\lambda}$ vanishes on $p$-singular elements of cycle shape $(n-r-p)(p)(1)^{r}$. This only leaves the values of $n$ listed in case (1).

Now we deal with the case that $V$ does not restrict irreducibly to $\mathcal{O A}_{n}$. Then $V$ is a simple $\mathcal{O S}_{n}$-module indexed by a self-conjugate partition (see e.g. []). Here we will show that unless we are in cases (2) or (3) the corresponding character $\chi_{\lambda}$ vanishes on some $p$-singular conjugacy class $C$ of $\mathfrak{S}_{n}$ contained in $\mathfrak{A}_{n}$ which forms a single $\mathfrak{A}_{n}$-class. Then both constituents of $\left.\chi_{\lambda}\right|_{\mathfrak{A}_{n}}$ will vanish on $C$, and again we are done by Corollary 2.3. 
Recall that a class of $\mathfrak{S}_{n}$ splits into two $\mathfrak{A}_{n}$-classes if and only if its elements have a cycle shape consisting of odd cycles of mutually distinct lengths. First assume that $n$ is odd and $r$ is even. If $\chi_{\lambda}$ belongs to an endotrivial module, then by Corollary 2.3 it cannot vanish on elements of cycle shapes $(n-p-2)(p)(1)^{2}$, and $(n-r)(r-2)(1)^{2}$ when $r>1$, respectively $(n-p-3)(p)(1)^{3}$ when $r=1$, which forces $\lambda$ to possess $n-p-2$-hooks and moreover either $n-r$ and $r-2$-hooks, or $n-p-3$-hooks. But there are no such self-conjugate partitions. Similarly, when $n$ is even and $r$ is odd, we look at the values on elements of cycle shapes $(n-r)(r-2)(2)$ (resp. $(n-2)(1)^{2}$ when $\left.r=2\right),(n-p-2)(p)(2)$, and $(n-p)(2),(2 p-2)(p)(2)$ when $r=0$, to see that there are no relevant self-conjugate $\lambda$.

If $n$ and $r$ are both even, the values on elements of type $(n-r)(r)$ for $r>0$, respectively of type $(n-p),(n-p-2)$ and $(n-p-3)(2)$ show that only $\lambda=\left(p, 2,1^{p-2}\right)$ can possibly index an endotrivial module, in which case moreover $n=2 p$. Finally, for $n$ and $r$ both odd, we argue with the cycle shapes $(n-r)(r-1)$ (for $r>1)$ respectively $((n-1) / 2)^{2}$, $(n-p-1)(1)^{p+1},(n-p-3)(p)(1)^{3}$ to see that necessarily $n=2 p+1$, and $\lambda$ must either be as in (3), or $p=5, \lambda=\left(4,3^{2}, 1\right)$, or $p=7$ and $\lambda=\left(4^{3}, 3\right)$. When $\lambda=\left(4,3^{2}, 1\right)$, then the corresponding character vanishes on elements of cycle shape $(5)(3)^{2}$, while for $\lambda=\left(4^{3}, 3\right)$, it vanishes on elements of cycle shape $(7)(6)(2)$. This completes the proof.

Proposition 4.3. The group $G=\mathfrak{A}_{n}$ does not have simple faithful endotrivial $k G$-modules for $k$ a field of characteristic 2 when $n \geq 8$.

Proof. We show that any irreducible character $\chi_{\lambda}$ of $\mathfrak{S}_{n}$ vanishes on some even order element contained in $\mathfrak{A}_{n}$ (which is sufficient since $\mathfrak{S}_{n}$-classes of even order elements never split considered as $\mathfrak{A}_{n}$-classes).

First assume that $n$ is even. For $n=8$ we have $\mathfrak{A}_{8}=\mathrm{L}_{4}(2)$, so the claim follows from Theorem [5.2, for $n=10$ it can be checked from the known character table of $\mathfrak{S}_{10}$. For $n \geq 12$ we use that $\mathfrak{A}_{n}$ contains elements of cycle shapes $(n-2)(2),(n-4)(4)$ and $(n-5)\left(2^{2}\right)$ to see that $\lambda$ necessarily has to possess hooks of lengths $n-2, n-4$ and $n-5$. Up to conjugates this only leaves $\lambda=(n-1,1),(n-2,2)$ and $(n-4,3,1)$. But the characters indexed by these partitions vanish on elements of cycle shapes $(n-5)\left(2^{2}\right)$, $(n-6)(6),(n-6)(6)$ respectively.

If $n$ is odd, then the cases $n=9,11$ can again be checked from the character tables. Now let $n \geq 13$. Then non-vanishing on elements of cycle shapes $(n-3)(2),(n-4)\left(2^{2}\right)$, and $(n-5)(4)$ implies that $\lambda$ has hooks of lengths $n-3, n-4$ and $n-5$, whence $\lambda=(n-1,1),\left(n-2,1^{2}\right)$ or $(n-4,4)$ up to conjugates. But the corresponding characters vanish on elements of cycle shapes $(n-3)(2),(n-5)(4),(n-7)(6)$ respectively.

Let us next consider the cyclic defect cases:

Proposition 4.4. Let $G=\mathfrak{A}_{n}$ with $5 \leq n \leq p<2 p$.

(a) If $n=p, p+1$ then $k G$ has no non-trivial simple endotrivial module.

(b) If $p+2 \leq n<2 p$ then $k G$ has exactly one non-trivial simple endotrivial module, namely the Specht module indexed by the partition $\left(p+1,1^{n-p-1}\right)$.

Proof. Let $P$ be a Sylow $p$-subgroup of $G$ and $H=N_{\mathfrak{A}_{n}}(P)$. By our assumptions on $n, P$ is cyclic of order $p$. Write $n=p+r$, with $0 \leq r<p$. Then $N_{\mathfrak{S}_{n}}(P) \cong\left(C_{p} \rtimes C_{p-1}\right) \times \mathfrak{S}_{r}$ 
and it follows that

$$
e=\left|H: C_{\mathfrak{A}_{n}}(P)\right|= \begin{cases}\frac{p-1}{2} & \text { if } n=p, p+1, \\ p-1 & \text { if } p+2 \leq n<2 p .\end{cases}
$$

Furthermore, an easy computation shows that

$$
|X(H)|= \begin{cases}\frac{p-1}{2} & \text { if } n=p, p+1, \\ p-1 & \text { if } p+2 \leq n<2 p .\end{cases}
$$

In both cases $|X(H)|=e$, meaning that all the indecomposable endotrivial modules lie in the principal block $\mathbf{B}_{0}$ of $\mathfrak{A}_{n}$. The Brauer tree of $\mathbf{B}_{0}$ is a straight line, with exceptional node sitting on one end in case $n=p, p+1$. Thus the claims in (a) and in the first part of (b) are a direct consequence of Theorem 3.7. It follows from the explicit knowledge of the Brauer tree that for $n \geq p+2$ the end node corresponds to the Specht module indexed by the hook partition $\left(p+1,1^{r-1}\right)$. This gives the remaining assertion in (b).

4.2. Faithful modules for $\tilde{\mathfrak{A}}_{n}$. We now discuss faithful simple $k G$-modules for $G=\tilde{\mathfrak{A}}_{n}$, $n \geq 5$, the double covering group of $\mathfrak{A}_{n}$, with center of order 2 .

We recall some facts from the ordinary representation theory of $\tilde{\mathfrak{S}}_{n}$, where $\tilde{\mathfrak{S}}_{n}$ denotes any of the two double covering groups of $\mathfrak{S}_{n}$. Let $\mathcal{D}(n)$ denote the set of partitions of $n$ into distinct parts. We say that a partition $\lambda$ is odd if its number of even parts is odd, and else we call it even.

The faithful complex irreducible characters of $\tilde{\mathfrak{S}}_{n}$ are parametrized by partitions $\lambda \in$ $\mathcal{D}(n)$ as follows: if $\lambda$ is even there is one irreducible character $\psi_{\lambda} \in \operatorname{Irr}\left(\tilde{\mathfrak{S}}_{n}\right)$ which splits upon restriction to $\tilde{\mathfrak{A}}_{n}$ into two distinct constituents $\psi_{\lambda}^{ \pm}$; if $\lambda$ is odd, there are two irreducible characters $\psi_{\lambda}^{ \pm} \in \operatorname{Irr}\left(\tilde{\mathfrak{S}}_{n}\right)$ which have the same restriction to $\tilde{\mathfrak{A}}_{n}$, see e.g. [25, Thm. 8.6].

Theorem 4.5. The group $G=\tilde{\mathfrak{A}}_{n}$ does not have faithful simple endotrivial $k G$-modules for $k$ a field of characteristic $p>0$ when $n \geq \min \{2 p, p+4\}$.

Proof. Note that $Z(G)$ lies in the kernel of any simple 2-modular $k G$-module, so we may certainly assume that $p>2$. Let first $\lambda \in \mathcal{D}(n)$ be odd and assume that $n \geq p+4$. Then the irreducible characters $\psi_{\lambda}^{ \pm}$of $\tilde{\mathfrak{S}}_{n}$ restrict irreducibly to $\tilde{\mathfrak{A}}_{n}$, so we may argue in $\tilde{\mathfrak{S}}_{n}$ by Lemma 2.2. Let $g \in \tilde{\mathfrak{S}}_{n}$ be an element whose projection to $\mathfrak{S}_{n}$ has cycle shape $\mu=(p)(2)(1)^{n-p-2}$. Then $\psi_{\lambda}^{ \pm}$vanishes on $g$ unless $\lambda=\mu$, by the theorem of Schur (see [25, Thm. 8.7(ii)]), while when $\lambda=\mu, \psi_{\lambda}^{ \pm}$vanishes on elements of cycle shape $(p)(4)(1)^{n-p-4}$. Since these elements are $p$-singular, $\psi_{\lambda}^{ \pm}$cannot be endotrivial by Corollary 2.3 . When $n=2 p<p+4$, so $p=3, n=6$, the only candidate is $\psi_{\lambda}^{ \pm}$with $\lambda=(3,2,1)$, and this vanishes on (3-singular) elements of cycle shape (6).

If $\lambda \in \mathcal{D}(n)$ is even, we need to look at the constituents $\psi_{\lambda}^{ \pm}$of the restriction of $\psi_{\lambda}$ to $\tilde{\mathfrak{A}}_{n}$. By [25, Thm. 8.7], $\psi_{\lambda}^{+}(g)=\psi_{\lambda}^{-}(g)$ whenever $g$ has cycle shape different from $\lambda$. Now elements with cycle shape $(p)(2)^{2}(1)^{n-p-4}$ are contained in $\tilde{\mathfrak{A}}_{n}$ and $\psi_{\lambda}$ vanishes on these again by [25, Thm. 8.7(iii)].

Note that faithful simple modules for the exceptional six-fold covering groups $6 . \mathfrak{A}_{6}$, $6 . \mathfrak{A}_{7}$ only exist in characteristic $p \geq 5$, so for covering groups of $\mathfrak{A}_{n}$ with centre of even 
order we are only left with cases with cyclic Sylow $p$-subgroup. We will deal with the exceptional covering groups in the next subsection.

Proposition 4.6. Let $G=\tilde{\mathfrak{A}}_{n}$ with $5 \leq p \leq n \leq p+3$. Then the faithful simple endotrivial $k G$-modules are precisely those indexed by the following partitions:

(1) $((p+1) / 2,(p-1) / 2)$ when $n=p$;

(2) $(p+1)$ and $((p+1) / 2,(p-1) / 2,1)$ when $n=p+1$;

(3) $(p+2)$ (two non-isomorphic modules) and, for $p>5,((p+1) / 2,(p-1) / 2,2)$ (two non-isomorphic modules) when $n=p+2$; and

(4) $(p+2,1)$ (two non-isomorphic modules) and, for $p>5,((p+1) / 2,(p-1) / 2,2,1)$ (two non-isomorphic modules) when $n=p+3$.

In characteristic $p=3, k \tilde{\mathfrak{A}}_{5}$ has two faithful simple endotrivial modules, both of dimension 2.

Proof. It is easily seen that for $5 \leq p \leq n \leq p+3$ the normalizer $H$ of a (cyclic) Sylow $p$ subgroup of $\mathfrak{A}_{n}$ has cyclic Sylow 2-subgroups, so the normalizer $\tilde{H}$ of a Sylow $p$-subgroup of $\tilde{\mathfrak{A}}_{n}$ has abelian Sylow 2-subgroups. In particular, $|X(\tilde{H})|=2|X(H)|$, and thus by Corollary [3.3(c) there exists exactly one non-trivial faithful $p$-block of $\tilde{\mathfrak{A}}_{n}$ containing simple endotrivial modules. (In contrast, for $n \geq p+4$ we have $|X(\tilde{H})|=|X(H)|$ and so there do not exist faithful simple endotrivial modules, in accordance with Theorem 4.5.)

First assume that $p \geq 5$. The Brauer trees for the faithful blocks of $\tilde{\mathfrak{A}}_{n}$ have been calculated by Müller [37. In our situation, there are five blocks to consider, corresponding to the $p$-bar cores ()$,(1),(2),(2,1)$, and (3). These have associated sign and $s$-invariant $(+, 0),(+, 1),(-, 2),(-, 2),(+, 1)$ respectively. By [37, Thm. 4.4] the Brauer tree is a straight line in the cases of sign "+", or with sign "-" and $p=5$, with the exceptional node at the end when $s=0$. Moreover, the end nodes are as given in the statement. Otherwise, the Brauer tree is a star with four arms of positive length and no exceptional node.

For the $p$-bar core (3), the degree of the character parametrized by the partition $(p+3)$ is twice the degree of the character for the $p$-bar core (2) parametrized by the partition $(p+2)$ (see $[25$, Thm. 10.7]) and hence not congruent to $\pm 1(\bmod p)$, whence this block cannot contain simple endotrivial modules. Thus, for $n=p+3$ the block with associated $p$-bar core $(2,1)$ is the one with simple endotrivial modules. Again the labels of the end nodes can be read off from [37, Thm. 4.4].

It ensues from [25, Thm. 10.7] that the dimensions of the endotrivial modules in Proposition 4.6 are given as follows, where $m:=(p-1) / 2$ :

\begin{tabular}{|c|lll|}
\hline$n$ & $\chi_{\lambda}(1)$ & & \\
\hline$p$ & $2^{m-1}\left(\begin{array}{c}p-1 \\
m\end{array}\right)$ & & \\
$p+1$ & $2^{m}$ & and & $2^{m}\left(\begin{array}{c}p-1 \\
m-2\end{array}\right)$ \\
$p+2$ & $2^{m}$ & and & $2^{m}\left(\begin{array}{c}p-1 \\
m-3\end{array}\right) \frac{(p+1)(p+2)}{p-1}$ \\
$p+3$ & $2^{m}(p+1)$ & and & $2^{m-1}\left(\begin{array}{c}p-1 \\
m-3\end{array}\right) \frac{(p-3)(p+2)}{3}$ \\
\hline
\end{tabular}

\subsection{The result for alternating groups.}


Lemma 4.7. Let $n=2 p+1>5$, and $V$ be a $k \mathfrak{A}_{n}$-constituent of the (irreducible) Specht module for $k \mathfrak{S}_{n}$ indexed by the partition $\lambda=\left(p+1,1^{p}\right)$. Then $V$ is endotrivial.

Proof. By [40, Thm. 2] the Specht module for $\lambda$ is irreducible modulo $p$. Let $H=\mathfrak{A}_{p+1} \times \mathfrak{A}_{p}$ denote a Young subgroup of $G=\mathfrak{A}_{n}$. Then $H$ contains a Sylow $p$-subgroup of $G$. Thus by Lemma 2.2 it suffices to show that the restriction $\left.V\right|_{H}$ of $V$ to $H$ is endotrivial. The Littlewood-Richardson rule shows that the ordinary character of $\left.V\right|_{H}$ is given by

$$
1 \otimes 1+\sum_{i=1}^{(p-1) / 2} \chi_{i} \otimes\left(\psi_{i}+\psi_{i+1}\right),
$$

where $\chi_{i}$ denotes the character indexed by the hook partition $\left(p+1-i, 1^{i}\right)$ and $\psi_{i}$ the character indexed by the hook partition $\left(p+1-i, 1^{i-1}\right)$. (Here, $\mathbb{\nabla}$ is the external tensor product.) The only constituent in the principal block is the trivial character, and all the $\chi_{i}$ are of defect zero for $\mathfrak{A}_{p+1}$, so sorting by blocks we get a direct decomposition

$$
\left.V\right|_{H}=k \oplus \bigoplus_{i=1}^{(p-1) / 2} P_{i} \otimes M_{i},
$$

where $P_{i}$ is the projective $k \mathfrak{A}_{p+1}$-module with character $\chi_{i}$ and $M_{i}$ is a $k \mathfrak{A}_{p}$-module with character $\psi_{i}+\psi_{i+1}$. It hence suffices to argue that all $M_{i}$ are projective $k \mathfrak{A}_{p}$-modules. We have already seen that $\left.V\right|_{\mathfrak{A}_{p+1}}=1+$ (projectives), so its restriction to $\mathfrak{A}_{p}<\mathfrak{A}_{p+1}$ also has this form. But the Young subgroup $\mathfrak{A}_{p}$ of $\mathfrak{A}_{p+1}$ is conjugate to the second factor of $H$, so we conclude that $\sum_{i} M_{i}$ is projective, and hence that each $M_{i}$ is.

This has also been shown in [10, Prop. 8.3] by a more involved argument.

Lemma 4.8. Let $n=3 p-1>5$, and $V$ be the $p$-modular reduction of the Specht module of $\mathfrak{S}_{n}$ indexed by the partition $\lambda=(2 p-1, p)$. Then $V$ is simple and endotrivial.

Proof. The Specht module for the partition $(2 p-1, p)$ is irreducible modulo $p$ by a theorem of Fayers and isomorphic to the Young module $Y^{(2 p-1, p)}$ (see [19, Prop. 1.1]). Restriction of $Y^{(2 p-1, p)}$ to the Young subgroup $\mathfrak{S}_{3 p-3}$ gives

$$
\left.Y^{(2 p-1, p)}\right|_{\mathfrak{S}_{3 p-3}}=Y^{(2 p-3, p)} \oplus 2 Y^{(2 p-2, p-1)}
$$

by two-fold application of [20, Thm. 5.1]. As $(2 p-2, p-1)$ is $p$-restricted, the Young module $Y^{(2 p-2, p-1)}$ is projective by [15. Thm. 2], while $Y^{(2 p-3, p)}$ is endotrivial by [10, Prop. 8.2]. The claim follows since $\mathfrak{S}_{3 p-3}$ contains a Sylow $p$-subgroup of $\mathfrak{S}_{3 p-1}$.

The proof of the endotriviality of $Y^{(2 p-1, p)}$ given in [10] seems unclear to us.

Theorem 4.9. Let $V$ be a faithful simple $k G$-module, for some covering group $G$ of $\mathfrak{A}_{n}$, $n \geq \max \{p, 5\}$, over a field $k$ of characteristic $p>0$. Then $V$ is endotrivial if and only if $V$ is a constituent of the simple module for the corresponding covering group of $\mathfrak{S}_{n}$ indexed by $\lambda \vdash n$, where one of:

(1) $G=\mathfrak{A}_{n}, 5 \leq p+2 \leq n<2 p$ and $\lambda=\left(p+1,1^{n-p-1}\right)$ (cyclic defect);

(2) $G=\tilde{\mathfrak{A}}_{n}, 3 \leq p \leq n \leq p+3$ and $\lambda$ is as in Proposition 4.6 (cyclic defect);

(3) $G=\mathfrak{A}_{n}, p>2, n=2 p+1$ and $\lambda=\left(p+1,1^{p}\right)$;

(4) $G=\mathfrak{A}_{n}, p>2, n=3 p-1$ and $\lambda=(2 p-1, p)$; or 
(5) $n=6,7,|Z(G)| \geq 3$ and $(G, p, V)$ are as in Table 1 .

Proof. By Theorem 4.5 and Proposition 4.6 the only examples with $|Z(G)|=2$ are those in (2) of the conclusion. Let us now assume that $G=\mathfrak{A}_{n}$. If $n<2 p$, the Sylow $p$-subgroups of $G$ are cyclic. Then Proposition 4.4 gives (1) of the conclusion.

So now assume that $G=\mathfrak{A}_{n}, n \geq 2 p$ and $p>2$. Then $V$ is an $\mathfrak{A}_{n}$-constituent of the $p$-modular reduction of the Specht module indexed by one of the partitions $\lambda \vdash n$ listed in Proposition 4.2. First consider the possibilities in case (1) of that result. Note that the $p$-core of these $\lambda$ is the partition $(r)$, so $V$ lies in the principal $p$-block. By James [28. Cor. 2.11] the only Specht module in the principal block which remains irreducible modulo $p$ is the trivial module, unless $n \equiv-1(\bmod p)$. Clearly the same holds for the module indexed by the conjugate partition, which is obtained by tensoring with the sign representation. Thus, we only need to consider the case $r=p-1, \lambda=(2 p-1, p)$. The corresponding Specht module is endotrivial by Lemma 4.8, leading to case (4). By the same criterion, the Specht module for $\lambda=\left(p, 2,1^{p-2}\right)$ as in Proposition 4.2(2) is reducible. Finally, the Specht module parametrized by the partition $\lambda=\left(p+1,1^{p}\right)$ in Proposition 4.2(3) is in fact an example by Lemma 4.7.

Now assume that $G=\mathfrak{A}_{n}$ and $p=2$. Then there are no examples for $n \geq 8$ by Proposition 4.3. For $\mathfrak{A}_{5}=\mathrm{L}_{2}(4)$, there's no example by Theorem [5.2, and for $n=6,7$, use of Corollary 2.3 and the character tables shows that no cases arise.

Finally, if $G$ is one of the 3 - or 6 -fold exceptional covering groups of $\mathfrak{A}_{6}$ or $\mathfrak{A}_{7}$, then the claim for $p=5,7$ follows by the cyclic defect methods from Section 3 , while for $p=2$ the character tables in [12] show that the only faithful candidate characters are those listed in Table 1, Explicit calculation with these modules yields that all candidates are in fact endotrivial.

TABLE 1. Relevant blocks in $6 \cdot \mathfrak{A}_{6}$ and $6 \cdot \mathfrak{A}_{7}$

\begin{tabular}{|r|r|r|c|c|l|}
\hline$G$ & $p$ & $X(H)$ & $X(H) / e$ & block & $\operatorname{dim} V$ \\
\hline \hline $3 . \mathfrak{A}_{6}$ & 2 & - & - & 4,5 & $3,3,9$ \\
\hline \hline $3 . \mathfrak{A}_{6}$ & 5 & 6 & 3 & 5,6 & 6 \\
$6 . \mathfrak{A}_{6}$ & 5 & 12 & 6 & 12,13 & 6,6 \\
\hline \hline $3 . \mathfrak{A}_{7}$ & 5 & 12 & 3 & 6,7 & 6,21 \\
$6 . \mathfrak{A}_{7}$ & 5 & 24 & 6 & 15,16 & $6,6,24$ \\
\cline { 2 - 6 } $3 . \mathfrak{A}_{7}$ & 7 & 9 & 3 & 6,7 & 6,15 \\
$6 . \mathfrak{A}_{7}$ & 7 & 18 & 6 & 15,16 & 6,6 \\
\hline
\end{tabular}

\section{Groups of LiE type in DEFINING CharaCteristiC}

In this section we classify the simple endotrivial modules for quasi-simple groups of Lie type in their defining characteristic. It turns out that these are extremely rare: they only occur for rank at most 2. Carlson, Mazza and Nakano [9] determined the structure of the group of endotrivial modules in these cases. Again, our results are independent and do not seem to follow in an obvious way from this. 
Let $G$ be a connected reductive linear algebraic group over the algebraic closure of a finite field of characteristic $p$. Let $\Phi$ denote the root system of $G$ with respect to some maximal torus, and $\Phi^{+} \subset \Phi$ a positive system. Let $\lambda_{1}, \ldots, \lambda_{l}$ denote the corresponding fundamental dominant weights of $G$, and $\rho=\sum_{i=1}^{l} \lambda_{i}$. We write $N:=\left|\Phi^{+}\right|$. For a dominant weight $\lambda$ of $G$ we denote by $L(\lambda)$ the corresponding simple highest weight module (see e.g. [35, $§ 15]$ ).

Proposition 5.1. Let $G$ be as above. Let $\lambda$ be a p-restricted dominant weight of $G$, different from the Steinberg weight $(p-1) \rho$. Then:

(a) $\operatorname{dim} L(\lambda)<p^{N}$.

(b) $\operatorname{dim} L(\lambda)<p^{N}-1$, unless $N=1$ and $\lambda=(p-2) \rho$.

(c) If $p=2$ then $\operatorname{dim} L(\lambda)<2^{N-1}-1$, unless $N \leq 3$.

Proof. By Weyl's character formula, the dimension of the corresponding highest weight module $L_{\mathbb{C}}(\lambda)$ for an algebraic group $G_{\mathbb{C}}$ of the same type over the complex numbers is given by

$$
\operatorname{dim} L_{\mathbb{C}}(\lambda)=\prod_{\alpha \in \Phi^{+}} \frac{\langle\lambda+\rho, \alpha\rangle}{\langle\rho, \alpha\rangle}
$$

(see [26, 24.3]). It is well-known that the dimension of $L_{\mathbb{C}}(\lambda)$ is an upper bound for the dimension of $L(\lambda)$. For $\lambda=(p-1) \rho$ the formula gives $\operatorname{dim} L_{\mathbb{C}}(\lambda)=\prod_{\alpha \in \Phi^{+}} p=p^{N}$. Since the Steinberg module $L_{\mathbb{C}}(\lambda)$ remains irreducible under restriction to characteristic $p$, this shows that $\operatorname{dim} L(\lambda)=p^{N}$. Any other $p$-restricted weight is of the form $\lambda=(p-1) \rho-\psi$, where $\psi=\sum_{i=1}^{l} a_{i} \lambda_{i} \neq 0$ is a non-negative integral linear combination of fundamental weights, with $a_{j}>0$, say. Then

$$
\begin{aligned}
\operatorname{dim} L(\lambda) \leq \operatorname{dim} L_{\mathbb{C}}(\lambda) & =\prod_{\alpha \in \Phi^{+}} \frac{\langle p \rho-\psi, \alpha\rangle}{\langle\rho, \alpha\rangle}=\prod_{\alpha \in \Phi^{+}}\left(p-\frac{\langle\psi, \alpha\rangle}{\langle\rho, \alpha\rangle}\right) \\
& \leq \prod_{\alpha \neq \alpha_{j}} p\left(p-\frac{\left\langle\psi, \alpha_{j}\right\rangle}{\left\langle\rho, \alpha_{j}\right\rangle}\right)=p^{N-1}\left(p-a_{j}\right),
\end{aligned}
$$

using that $\langle$,$\rangle is linear in the first argument. Clearly, this is smaller than p^{N}-1$ unless $N=1, \lambda=(p-2) \lambda_{1}$, which gives (a) and (b).

If $p=2$, then the above argument shows that $\operatorname{dim} L(\lambda) \leq 2^{N-1}$ when $\lambda \neq \rho$. If $N>1$ then since $\Phi$ is indecomposable there is at least one further positive root $\alpha_{j}+\alpha_{m} \in \Phi^{+}$ which involves $\alpha_{j}$. Then we get

$$
\operatorname{dim} L(\lambda) \leq 2^{N-2}(2-1)\left(2-\frac{1}{2}\right)=3 \cdot 2^{N-3},
$$

which is smaller than $2^{N-1}-1$ when $N>3$.

We thank Frank Lübeck for showing us the proof of (a).

Theorem 5.2. Let $G$ be a finite quasi-simple group of Lie type in characteristic $p>0$. Let $V$ be a simple faithful $k G$-module, where $k$ is algebraically closed of characteristic $p$. Then $V$ is endotrivial if and only if one of

(1) $p \geq 5, G=\mathrm{SL}_{2}(p)$ and $\operatorname{dim} V=p-1$; or

(2) $p=2, G=\mathrm{SL}_{3}(2)$ and $\operatorname{dim} V=3$. 
Proof. Note that the exceptional Schur multipliers of groups of Lie type all have order a power of the defining characteristic $p$, so lie in the kernel of all $k G$-representations. Thus, we may assume that $V$ is a (not necessarily faithful) non-trivial simple $k H$-module, where $H$ is a group of simply connected type such that $G=H / Z$ for some central subgroup $Z \leq H$.

According to Steinberg's tensor product theorem (see e.g. [35, Thm. 16.12]) the simple $k \mathrm{H}$-modules are tensor products of Frobenius twists of $p$-restricted highest weight modules. If any of the factors in such a tensor product is a twist of the Steinberg module, then $\operatorname{dim} V$ is divisible by $p$, hence not endotrivial by Lemma 2.1. All other $p$-restricted highest weight modules have dimension at most $p^{N}-1$, and even strictly smaller than this unless $H$ is of type $A_{1}$, by Proposition 5.1. Thus $V$ has dimension smaller than $q^{N}-1$, where $|H|_{p}=q^{N}$, unless $H$ is of type $A_{1}$ and $p=q$. In the former case $V$ cannot be endotrivial by Lemma 2.1 for $p>2$.

If $p=2$ then either $N=3$, in which case $H=\mathrm{SL}_{3}(2)$ (note that $\mathrm{SU}_{3}(2)$ is solvable), or $\operatorname{dim} V<2^{N-1}-1$ by Proposition 5.1. As $|H|_{2}=2^{N}$, the latter modules cannot be endotrivial by Lemma 2.1. The natural representation of $\mathrm{SL}_{3}(2) \cong \mathrm{L}_{2}(7)$ is an example by Proposition 3.8.

So finally assume that $H=\mathrm{SL}_{2}(p)$ and $\operatorname{dim} V=p-1$. Let $P \leq H$ denote a Sylow $p$-subgroup. Since $\left.V\right|_{P}$ is indecomposable, and up to isomorphism there is a unique indecomposable $k P$-module of dimension $p-1$, namely $\Omega(k)$, we must have $\left.V\right|_{P} \cong \Omega(k)$, which is endotrivial. Thus this is indeed an endotrivial module by Lemma 2.2 ,

\section{Groups of Lie type in CROSS CharacteristiC}

In this section we investigate simple endotrivial modules for groups of Lie type in nondefining characteristic $\ell$. Here our results are not complete. Throughout this section let $k$ denote a large enough field of characteristic $\ell$. We refer to the book of Carter [11] for notation and background.

6.1. Auxiliary results. We first prove some general criteria to rule out endotriviality of certain modules.

Our first observation makes use of Harish-Chandra theory. Let $G$ be a finite group with a split BN-pair of characteristic $p \neq \ell$. Let $Q \leq G$ be a parabolic subgroup, with Levi decomposition $Q=U . L$, so that $U$ is a normal $p$-subgroup of $Q$ with complement $L$. Let $\tilde{V}$ be a $\mathbb{C} G$-module with character $\chi$, and $V$ the $\ell$-modular reduction of a suitable lattice in $\tilde{V}$. The restriction of $\tilde{V}$ to $Q$ decomposes into a direct sum $\left.\tilde{V}\right|_{Q}=\tilde{V}^{U} \oplus \tilde{V}^{\prime}$, where no constituent of $\tilde{V}^{\prime}$ has $U$ in its kernel. As customary, we'll write ${ }^{*} R_{L}^{G}(\tilde{V})$ for the $L$-module $\tilde{V}^{U}$ of $U$-fixed points, the Harish-Chandra restriction of $\tilde{V}$. Since all $\ell$-modular constituents of ${ }^{*} R_{L}^{G}(\tilde{V})$ have $U$ in their kernel, and none of the $\ell$-modular constituents of $\tilde{V}^{\prime}$ have, these two summands lie in different $\ell$-blocks of $G$, and so there is a corresponding decomposition of $\left.V\right|_{Q}$ which (by abuse of notation!) we write $\left.V\right|_{Q}={ }^{*} R_{L}^{G}(V) \oplus V^{\prime}$. Thus we have:

Lemma 6.1. In the above setting, assume that $V$ is endotrivial. Then exactly one of ${ }^{*} R_{L}^{G}(V), V^{\prime}$ is endotrivial, and the other is projective. In particular, one has dimension congruent to $\pm 1\left(\bmod |L|_{\ell}\right)$, and the other has dimension divisible by $|L|_{\ell}$. 
For finite groups of Lie type, the Harish-Chandra restriction of ordinary irreducible characters can be computed from information inside relative Weyl groups. In order to formulate this, we introduce the following setup. Let $\mathbf{G}$ be a simple algebraic group of simply connected type over an algebraic closure of a finite field $\mathbb{F}_{p}$, and $F: \mathbf{G} \rightarrow \mathbf{G}$ a Steinberg endomorphism, with finite group of fixed points $G=\mathbf{G}^{F}$. Let $\mathbf{G}^{*}$ be a Langlands dual group to $\mathbf{G}$, with corresponding Steinberg map also denoted by $F$. We write $q$ for the absolute value of the eigenvalues of $F$ on the character group of an $F$-stable maximal torus of $\mathbf{G}$ (this is a power of $p$, integral unless $G$ is of Ree or Suzuki type). We then also sometimes write $G=G(q)$. Now Lemma 6.1 leads to the following:

Lemma 6.2. Let $G=G(q)$ be a quasi-simple group of Lie type and $\chi \in \operatorname{Irr}(G)$. Assume that $V$ is a simple endotrivial $k G$-module, for $k$ a field of characteristic $\ell$ dividing $q-1$, whose Brauer character is the restriction of $\chi$ to $\ell$-classes.

(a) Then $\chi$ lies in the Harish-Chandra series of some (linear) character $\theta$ of a maximally split torus $T$ of $G$; let $\psi \in \operatorname{Irr}\left(W_{G}(T, \theta)\right)$ denote the corresponding irreducible character of the relative Weyl group $W_{G}(T, \theta):=N_{G}(T, \theta) / T$.

(b) We have either $\chi(1) \equiv \psi(1) \equiv \pm 1\left(\bmod |T|_{\ell}\right)$ or $\psi(1) \equiv 0\left(\bmod |T|_{\ell}\right)$.

Proof. By Lemma 2.1 we know that $\chi(1) \equiv \pm 1\left(\bmod |G|_{\ell}\right)$; in particular, $\chi$ is of $\ell$-height zero. The first claim is then a consequence of [34, Thm. 7.5].

Let $B$ be a Borel subgroup containing $T$, with unipotent radical $U$. By Lemma 6.1, $\left.V\right|_{B}={ }^{*} R_{T}^{G}(V) \oplus W$ for some $k B$-module $W$, and one of ${ }^{*} R_{T}^{G}(V), W$ is projective. If ${ }^{*} R_{T}^{G}(V)$ is projective then on the level of characters this means that ${ }^{*} R_{T}^{G}(\chi)(1) \equiv 0$ $\left(\bmod |T|_{\ell}\right)$, while if ${ }^{*} R_{T}^{G}(V)$ is endotrivial, then ${ }^{*} R_{T}^{G}(\chi)(1) \equiv \pm 1\left(\bmod |T|_{\ell}\right)$, where the sign is the same as for $\chi$.

Now assume that $\chi$ lies in the Harish-Chandra series of the character $\theta$ of $T$ and corresponds to $\psi \in \operatorname{Irr}\left(W_{G}(T, \theta)\right)$. Then we have ${ }^{*} R_{T}^{G}(\chi)=\psi(1) \theta$, from which we may conclude since $\theta$ is a linear character.

For our second approach recall Lusztig's partition $\operatorname{Irr}(G)=\coprod_{s} \mathcal{E}(G, s)$ of the irreducible characters of $G$ into disjoint Lusztig series, indexed by a system of representatives $s$ of the semisimple conjugacy classes of the dual group $G^{*}=\mathbf{G}^{* F}$.

For a prime $\ell$ not dividing $q$ we let $d_{\ell}(q)$ denote the multiplicative order of $q$ modulo $\ell$, respectively $d_{2}(q):=2$ when $\ell=2$ and $q \equiv 3(\bmod 4)$. We need the following result:

Proposition 6.3. Let $\chi \in \mathcal{E}(G, s)$ be the character of a faithful simple endotrivial $k G$ module, where $k$ is algebraically closed of characteristic $\ell$. Then $s$ lies in some maximal torus $T \leq G^{*}$ containing a Sylow $d_{\ell}(q)$-torus of $G^{*}$.

Proof. Observe that endotrivial modules are of height zero, by Lemma 2.1. By [34, Prop. 7.2] any irreducible character of $G$ of $\ell$-height zero lies in a Lusztig series $\mathcal{E}(G, s)$ where $C_{G^{*}}(s)$ contains a Sylow $\ell$-subgroup of $G^{*}$. By [34, Thm. 5.9] such elements lie in a maximal torus of $G^{*}$ containing a Sylow $d_{\ell}(q)$-torus $S^{*}$.

The following vanishing result (proved for example in [18, Lem. 3.2]) will be crucial:

Proposition 6.4. Let $x \in G$ be semisimple and $\chi \in \mathcal{E}(G, s)$ with $\chi(x) \neq 0$. Then there exists a maximal torus $T \leq G$ with $x \in T$, and such that $T^{*} \leq C_{G^{*}}(s)$ for a torus $T^{*} \leq G^{*}$ in duality with $T$. 
6.2. The case $\ell=2$. We first show that no new examples arise for $\ell=2$ and thus prove Theorem 1.2(a) of the introduction.

Here we use the well-known fact that non-trivial self-dual simple modules in characteristic 2 have even dimension. This implies in particular:

Corollary 6.5. Let $G$ be a group all of whose elements are real (i.e., conjugate to their inverse). Then $G$ cannot have non-trivial simple endotrivial modules in characteristic 2.

Proof. Observe that any complex character $\chi$ of $G$ is real valued, since its complex conjugate satisfies $\bar{\chi}(g)=\chi\left(g^{-1}\right)=\chi(g)$ by assumption. If $V$ is a simple endotrivial module for $G$ in characteristic 2, then by Theorem 1.3 it is the 2-modular reduction of a simple $\mathbb{C} G$-module, which is self-dual since its character is real. But then $V$ is self-dual and thus either trivial or of even dimension; the latter being excluded by Lemma 2.1 .

Proposition 6.6. Let $G$ be a finite simple group of Lie type. Let $\chi \neq 1$ be a unipotent character of $G$ whose 2-modular reduction $\chi^{0}$ is irreducible. Then $\chi^{0}$ is not endotrivial.

Proof. For groups defined over a field of characteristic 2, this was shown in Theorem 5.2 (note that by [11, $\S 13]$ the 3-dimensional characters of $\mathrm{SL}_{3}(2)$ are not unipotent). So now assume that $G$ is defined in odd characteristic. Let $\chi$ be a unipotent complex character of $G$. First assume that $\chi$ is uniquely determined by its multiplicities in the Deligne-Lusztig characters. Since the latter are rational valued, $\chi$ is also rational and thus self-dual. Hence the same is true for its 2-modular reduction. So $\chi^{0}(1)$ is even by the previous remark, and $\chi^{0}$ cannot be endotrivial.

Now by the fundamental results of Lusztig if $G$ is a classical group, then all unipotent characters do have the above property. For $G$ of exceptional type, the only unipotent characters not determined by their multiplicities are those for which the associated eigenvalue of Frobenius is non-real. These are necessarily not in the principal series, and an easy check shows that all of them have even degree, whence we may conclude as before.

Theorem 6.7. Let $G$ be a finite quasi-simple group. Then $G$ has a non-trivial simple endotrivial $k G$-module $V$ over a field of characteristic 2 if and only if one of:

(a) $G=\mathrm{L}_{2}(q)$ with $7 \leq q \equiv 3(\bmod 4)$ and $\operatorname{dim}(V)=(q-1) / 2$; or

(b) $G=3 . \mathfrak{A}_{6}$ and $\operatorname{dim}(V) \in\{3,9\}$.

In particular, Conjecture 1.1 holds for the prime 2.

Proof. We go through the various possibilities for $G$ according to the classification. If $G$ is alternating, the claim is Theorem 4.9, for $G$ sporadic there are no non-trivial simple endotrivial modules in characteristic 2 by Theorem 7.1 below. If $G$ is of Lie type over a field of even order, the endotrivial simple modules were obtained in Theorem 5.2. If $G$ is an exceptional group of Lie type in odd characteristic, the claim will follow from Theorems 6.8 and 6.11 below. Thus we only need to deal with classical groups of Lie type. Moreover, by Proposition 6.6 we may assume that $V$ is not the reduction of a unipotent representation.

First let $G=\operatorname{SL}_{n}(q)$ with $q$ odd. By Proposition 3.8 we may assume that $n \geq 3$. For $n=3$ it follows from the explicitly known character table [17] that among the complex irreducible characters the only candidates have dimension $q\left(q^{2}+q+1\right)$, and $q \equiv 3$ $(\bmod 4)$. But by $[23$, App.] these characters do not remain irreducible modulo 2. For 
$n=4$, the only non-unipotent complex irreducible characters of odd degree are of degrees $\Phi_{3} \Phi_{4} / 2, q^{2} \Phi_{3} \Phi_{4} / 2$ and lie in the Lusztig series of semisimple elements with centralizer $\mathrm{GL}_{2}\left(q^{2}\right) \cdot 2 /(q-1)$ in the dual group. (Here, and later, we write $\Phi_{d}$ for the $d$ th cyclotomic polynomial evaluated at $q$.) They satisfy the congruence in Lemma 2.1 when $q \equiv 3$ $(\bmod 4)$. But then there exist elements of order $2 \Phi_{4}$ in $G / Z(G)$ (in a torus of order $\left.\left(q^{4}-1\right) /(q-1)\right)$, while both characters are of $\Phi_{4}$-defect zero.

Now assume that $n \geq 5$. By Proposition 6.3, $V$ is the reduction of an ordinary representation lying in the Lusztig series of a semisimple element $s$ of $G^{*}=\operatorname{PGL}_{n}(q)$ contained in the centralizer of a Sylow $d$-torus, with $d \in\{1,2\}$. These have order $\Phi_{1}^{n-1}$, $\left(\Phi_{1} \Phi_{2}\right)^{\lfloor(n-1) / 2\rfloor}\left(\Phi_{1}\right)^{\delta}$ respectively, with $\delta \in\{0,1\}$. Thus, the centralizers of elements in such tori are contained in centralizers of $\Phi_{1^{-}}$or $\Phi_{2}$-tori, so are (images under the natural map $\left.\mathrm{GL}_{n}(q) \rightarrow G^{*}\right)$ of products of $\operatorname{groups} \mathrm{GL}_{m}(q)$ and $\mathrm{GL}_{m}\left(q^{2}\right)$. If $n$ is odd, a maximal torus of type $(n-1)(1)$ of $G^{*}$ contains regular elements of even order in $\left[G^{*}, G^{*}\right]$, so $\left|C_{G^{*}}(s)\right|$ must be divisible by a primitive prime divisor of $q^{n-1}-1$. This is only the case for $\mathrm{GL}_{n-1}(q)$ and $\mathrm{GL}_{(n-1) / 2}\left(q^{2}\right)$. But there also exist regular elements in maximal tori of type $(n-2)(2)$ and as none of the candidate groups contains such a torus, we are done by Proposition 6.4. If $n$ is even, we argue similarly that no centralizer contains maximal tori of types $(n-2)(2)$ and $(n-3)(3)$.

Next assume that $G=\mathrm{SU}_{n}(q)$ with $q$ odd, $n \geq 3$. When $n=3$, [17] shows that the only candidates are characters of degree $q \Phi_{6}$ when $q \equiv 1(\bmod 4)$, but again these are not irreducible mod 2. For $n=4$, the only candidates are of degree $\Phi_{4} \Phi_{6} / 2, q^{2} \Phi_{4} \Phi_{6} / 2$, with $q \equiv 1(\bmod 4)$. Again, these vanish on elements of order $2 \Phi_{4}$ of $G / Z(G)$. Finally, for $n \geq 5$ we may argue as for $\mathrm{SL}_{n}(q)$, replacing all tori by their Ennola duals, with order obtained by simply replacing $q$ by $-q$.

If $G$ is of type $B_{n}, C_{n}$ or $D_{2 n}$, then the Sylow $d$-tori of $G^{*}$ are maximal tori, hence self-centralizing, and the only elements $s$ centralizing a Sylow 2-subgroup are involutions. By [17] no examples arise for $G=\operatorname{Sp}_{4}(q)$. Now assume that $G=\operatorname{Spin}_{2 n+1}(q), n \geq 3$. Recall that the $G$-conjugacy classes of maximal tori are parametrized by conjugacy classes in the Weyl group (see e.g. [35, Prop. 25.1]), which in turn are naturally indexed by pairs of partitions of $n$. The maximal tori of $G^{*}$ of type $((1),(n-1))$ contain regular elements of even order, thus $C_{G}(s)$ is of type $C_{1} C_{n-1}$, or ${ }^{2} A_{n}$ when $n$ is even. On the other hand, neither of these contains a maximal torus of type $(-,(n-2)(2))$, so we may conclude by Proposition 6.4. Next take $G=\operatorname{Sp}_{2 n}(q), n \geq 3$. Again arguing with the maximal torus of type $((1),(n-1))$, we are left with the possible centralizers of type $B_{n-1},{ }^{2} D_{n-1} B_{1}, D_{n}$ and ${ }^{2} D_{n}$. Only the one of type $D_{n}$ contains maximal tori of type $(-,(n-2)(2))$, but it does not contain a maximal torus of type $((2),(n-2))$.

Next let $G=\operatorname{Spin}_{2 n}^{+}(q), n \geq 4$. Arguing with the maximal tori of type $(-,(n-1)(1))$ we see that only characters in Lusztig series parametrized by involutions with centralizer of type ${ }^{2} D_{n-1}$, or ${ }^{2} A_{n-1}$ with $n$ even, matter. But these do not contain tori of type $(-,(n-2)(2))$. The maximal tori in type ${ }^{2} D_{n}$ for $n$ odd are just Ennola dual to those in type $D_{n}$, and then an analogous argument deals with this case. Finally, all elements in $\operatorname{Spin}_{4 n}^{-}(q), n \geq 2$, are real by [46, Thm. 1.2], so no examples arise by Corollary 6.5.

6.3. Small rank exceptional groups. We now consider in more detail the exceptional groups of Lie type, and first treat the five families of small rank, that is, the Suzuki and 
Ree groups ${ }^{2} B_{2}\left(2^{2 f+1}\right),{ }^{2} G_{2}\left(3^{2 f+1}\right)$ and ${ }^{2} F_{4}\left(2^{2 f+1}\right)$, and the groups $G_{2}(q)$ and ${ }^{3} D_{4}(q)$. For all of these complete ordinary character tables are available, which makes it relatively easy to find the candidates for simple endotrivial modules in these cases.

Theorem 6.8. Let $G$ be a covering group of one of the simple groups ${ }^{2} B_{2}\left(2^{2 f+1}\right)$ (with $f \geq 1$ ), ${ }^{2} G_{2}\left(3^{2 f+1}\right.$ ) (with $f \geq 1$ ), $G_{2}(q)$ (with $q \geq 3$ ), ${ }^{3} D_{4}(q)$, or ${ }^{2} F_{4}\left(2^{2 f+1}\right.$ ) (with $f \geq 1$ ). Let $\ell \neq p$ denote a prime divisor of $|G|$ and $P$ a Sylow $\ell$-subgroup of $G$.

(a) If there exists a non-trivial simple endotrivial $k G$-module then $P$ is cyclic.

(b) The simple endotrivial $k G$-modules for primes $\ell$ such that $P$ is cyclic are precisely as given in Tables 2 and 3 (where $\ell \mid \Phi_{d}$ ).

TABLE 2. Simple endotrivial modules for low rank exceptional groups

\begin{tabular}{|r|r|r|c|c|c|}
\hline$G$ & $d$ & $|X(H)|$ & $|X(H)| / e$ & block & se $(\mathbf{B})$ \\
\hline \hline${ }^{2} B_{2}\left(q^{2}\right)$ & $1^{\prime}$ & 2 & 1 & 1 & 2 \\
${ }^{2} B_{2}\left(q^{2}\right)$ & $8^{\prime}$ & 4 & 1 & 1 & 3 \\
${ }^{2} B_{2}\left(q^{2}\right)$ & $8^{\prime \prime}$ & 4 & 1 & 1 & 3 \\
\hline \hline${ }^{2} G_{2}\left(q^{2}\right)$ & $1^{\prime}$ & 4 & 2 & 1,2 & 2 \\
${ }^{2} G_{2}\left(q^{2}\right)$ & 4 & 6 & 1 & 1 & 5 \\
${ }^{2} G_{2}\left(q^{2}\right)$ & $12^{\prime}$ & 6 & 1 & 1 & 5 \\
${ }^{2} G_{2}\left(q^{2}\right)$ & $12^{\prime \prime}$ & 6 & 1 & 1 & 5 \\
\hline \hline$G_{2}(q)$ & 3 & 6 & 1 & 1 & 4 \\
$G_{2}(q)$ & 6 & 6 & 1 & 1 & 4 \\
\hline \hline${ }^{3} D_{4}(q)$ & 12 & 4 & 1 & 1 & 2 \\
\hline \hline${ }^{2} F_{4}\left(q^{2}\right)$ & 12 & 6 & 1 & 1 & 4 \\
${ }^{2} F_{4}\left(q^{2}\right)$ & $24^{\prime}$ & 12 & 1 & 1 & 8 \\
${ }^{2} F_{4}\left(q^{2}\right)$ & $24^{\prime \prime}$ & 12 & 1 & 1 & 8 \\
\hline
\end{tabular}

Here $\Phi_{1}^{\prime}=q^{2}-1, \Phi_{8}^{\prime}=q^{2}+\sqrt{2} q+1, \Phi_{8}^{\prime \prime}=q^{2}-\sqrt{2} q+1, \Phi_{12}^{\prime}=q^{2}+\sqrt{3} q+1$, $\Phi_{12}^{\prime \prime}=q^{2}-\sqrt{3} q+1, \Phi_{24}^{\prime}=q^{4}+\sqrt{2} q^{3}+q^{2}+\sqrt{2} q+1, \Phi_{24}^{\prime \prime}=q^{4}-\sqrt{2} q^{3}+q^{2}-\sqrt{2} q+1$.

TABLE 3. Simple endotrivial modules for exceptional covering groups

\begin{tabular}{|r|r|r|c|r|}
\hline$G$ & $d$ & $|X(H)|$ & $|X(H)| / e$ & $\operatorname{dim} V$ \\
\hline \hline $2 .{ }^{2} B_{2}(8)$ & 5 & 8 & 2 & 56,56 \\
$2 .{ }^{2} B_{2}(8)$ & 7 & 4 & 2 & 64 \\
$2 .{ }^{2} B_{2}(8)$ & 13 & 8 & 2 & 40,40 \\
\hline \hline $3 . G_{2}(3)$ & 7 & 18 & 3 & $27,27,351,351,729(2 \times$ each $)$ \\
$3 . G_{2}(3)$ & 13 & 18 & 3 & $27,27,378,378,729(2 \times$ each $)$ \\
\hline \hline $2 . G_{2}(4)$ & 7 & 12 & 2 & 104,104 \\
$2 . G_{2}(4)$ & 13 & 12 & 2 & 12,1260 \\
\hline
\end{tabular}


Proof. We first deal with the case that $G$ itself is simple. First assume that $P$ is cyclic. It turns out that $\left|X\left(N_{G}(P)\right)\right| / e=1$ in all cases except for ${ }^{2} G_{2}\left(q^{2}\right)$ with $\ell \mid q^{2}-1$, so that by Lemma 3.2 the only $\ell$-block containing simple endotrivial modules is the principal block. The position of endotrivial modules on the Brauer tree is then described by Theorem 3.7. The Brauer trees for these groups have been determined by Hiß [22]. From this, the results in Table 2 follow. For the case of ${ }^{2} G_{2}\left(q^{2}\right)$ with $\ell \mid q^{2}-1$ we obtain $\left|X\left(N_{G}(P)\right)\right| / e=2$, and again the corresponding Brauer trees can be found in [22].

So now assume that $P$ is not cyclic. Note that all Sylow $\ell$-subgroups of ${ }^{2} B_{2}\left(2^{2 f+1}\right)$, for $\ell \neq 2$, are cyclic, so there is nothing to prove for these groups. First assume that $G={ }^{2} G_{2}\left(q^{2}\right)$, with $q^{2}=3^{2 f+1}$. The only prime $\ell \neq 3$ for which the Sylow $\ell$-subgroups of $G$ are not cyclic is $\ell=2$. From the known generic character table (see Chevie [17]) it is easy to check that only one non-trivial complex irreducible character $\chi$, of degree $q^{4}-q^{2}+1$, has the property that its value on involutions is of absolute value 1 . But from the known decomposition matrix of $G$ in [31] it follows that this character does not remain irreducible modulo 2 .

Next assume that $G=G_{2}(q)$ with $q>2$. The relevant primes $\ell$ in this case are exactly the prime divisors of $q^{2}-1$. From the character tables in [17] one sees that all non-trivial irreducible characters $\chi \in \operatorname{Irr}(G)$ satisfy $|\chi(g)|^{2} \neq 1$ on 2 -central involutions (when $p \neq 2$ ) and on 3 -central elements (when $p \neq 3$ ), so we may assume that $\ell \geq 5$ divides exactly one of $q-1$ or $q+1$. In both cases, the non-trivial characters of degree congruent to \pm 1 $(\bmod \ell)$ are seen to take values either 0 or of absolute value bigger than 1 on suitable $\ell$-singular elements.

For $G={ }^{3} D_{4}(q)$ the relevant primes are the prime divisors of $q^{6}-1$. The argument is now completely parallel to the one for $G_{2}(q)$ above, using the generic character tables.

Finally assume that $G={ }^{2} F_{4}\left(q^{2}\right)$ with $q^{2}=2^{2 f+1} \geq 8$. Here, the relevant primes are the prime divisors of $q^{8}-1$. We may argue as in the previous cases, using the generic character table in [17]. The only remaining candidates occur for $\ell=5$. They are characters of degree $\left(q^{4}-1\right)\left(q^{4}-q^{2}+1\right)\left(q^{12}+1\right)\left(q^{2}+\sqrt{2} q+1\right)$ when $f \equiv 1,2(\bmod 4)$, or $\left(q^{4}-1\right)\left(q^{4}-q^{2}+1\right)\left(q^{12}+1\right)\left(q^{2}-\sqrt{2} q+1\right)$ when $f \equiv 0,3(\bmod 4)$. But according to [21, Tables C3 and C4], these characters are reducible modulo $\ell$. (They are denoted $\chi_{8,1}$ respectively $\chi_{10,1}$ in loc. cit.)

The only proper covering groups in our situation are the groups $2 .{ }^{2} B_{2}(8), 3 . G_{2}(3)$ and $2 . G_{2}(4)$. When the Sylow $p$-subgroups are cyclic, we may conclude by using the criteria in Theorem 3.7 and information on the Brauer trees. The Sylow $p$-subgroups of the groups in question are non-cyclic only for $p \leq 5$ for $2 . G_{2}(4)$, respectively $p \leq 3$ for $2 .{ }^{2} B_{2}(8)$ and $3 . G_{2}(3)$. The ordinary character tables are known for all of these groups and the usual criteria give the claim.

6.4. Exceptional groups of large rank. We now turn to the exceptional groups of rank at least four, for which no complete generic character tables are available. We obtain an almost complete picture for unipotent characters:

Proposition 6.9. Let $G$ be a finite simple exceptional group of Lie type in characteristic $p$ of rank at least 4 and $\ell \neq p$ a prime for which the Sylow $\ell$-subgroups of $G$ are noncyclic. Then the candidates for non-trivial unipotent characters with endotrivial $\ell$-modular reduction are given in Table 4 . 
TABLE 4. Candidates for endotrivial unipotent characters

\begin{tabular}{|r|r|r|l|}
\hline$G$ & $d$ & $\ell$ & $\chi$ \\
\hline \hline$F_{4}$ & 4 & 5 & $F_{4}^{I I}[1]$ \\
$E_{6}$ & 4 & 5 & $D_{4}, r, \phi_{80,7}$ \\
$E_{6}$ & 6 & 19 & $\phi_{6,25}$ \\
${ }^{2} E_{6}$ & 4 & 5 & ${ }^{2} E_{6}[1], \phi_{16,5}$ \\
$E_{8}$ & 10 & 31 & $\phi_{28,68}$ \\
\hline
\end{tabular}

Here, the notation for unipotent characters is as in [11, §13].

Proof. First note that we may assume $\ell \neq 2$ by Proposition 6.6. Also, the Steinberg character is not endotrivial. Indeed, since we assume that the Sylow $\ell$-subgroups of $G$ are non-cyclic, there exist $\ell$-elements $g \in G$ with centralizer of positive semisimple rank. Then $C_{G}(g)$ contains unipotent elements, and thus there exist $p$-singular elements in $G$ of order divisible by $\ell$. But the Steinberg character takes value 0 on all $p$-singular elements, which shows that it cannot be endotrivial by Corollary 2.3 .

The degrees of the unipotent characters of groups of Lie type are known; they can be found in [11, §13] or in [17], for example. Let $d:=d_{\ell}(q)$ where $G=G(q)$. The condition that the Sylow $\ell$-subgroups are non-cyclic forces $\Phi_{d}$ to divide the order polynomial of $G$ at least twice, which restricts the possible values of $d$. For each type and each such $d$, we use the following criteria to eliminate candidates:

- we have $\chi(1) \not \equiv 0\left(\bmod \Phi_{d}(q)\right)$;

- if $d=1$ then $\chi(1)$ has to satisfy the congruence in Lemma 6.2(b);

- the Harish-Chandra restriction of $\chi$ to proper Levi subgroups $L$ of $G$ must satisfy the congruences in Lemma 6.1; and

- we have $\chi(1) \equiv \pm 1\left(\bmod |G|_{\ell}\right)$.

At this stage, usually only very few characters are left, which are then handled by ad hoc methods. We give some indications for $G=F_{4}(q)$. For $d=1,3,6$, no candidates remain. For $d=2$, we are left with the two cuspidal unipotent characters denoted $F_{4}^{I I}[1]$ and $F_{4}[-1]$, with $\ell=11$ respectively $\ell=5$. These are of defect zero for a Zsigmondy prime divisor (see [27, Thm. IX.8.3]) $r$ of $\Phi_{6}$ respectively $\Phi_{3}$. Since $B_{4}(q) \leq F_{4}(q)$ contains maximal tori of orders $\Phi_{1} \Phi_{2} \Phi_{3}$ and $\Phi_{1} \Phi_{2} \Phi_{6}$ (parametrized by the pairs of partitions $((3),(1))$ and $((1),(3)))$ there exist elements of order $r \ell$ in $G$, so these characters cannot be endotrivial by Corollary 2.3. Note that $q \neq 2$ when $\ell=11 \mid(q+1)$, so there does exist a Zsigmondy prime for $\Phi_{6}$ in our situation. For $d=4$, there remain the cuspidal unipotent characters $F_{4}[ \pm i]$ and $F_{4}^{I I}[1]$ with $\ell=5$. The character $F_{4}^{I I}[1]$ occurs in our list, while the characters $F_{4}[ \pm i]$ are of 3-defect zero, and $G$ contains elements of order 15.

For $G=E_{6}(q)$, no candidates remain for $d=1,3$. For $d=2$, all remaining candidates are of $\Phi_{5^{-}}$or $\Phi_{8}$-defect zero, so do not lead to examples as $G$ contains tori of order $\Phi_{1} \Phi_{2} \Phi_{5}$ and $\Phi_{1} \Phi_{2} \Phi_{8}$. For $d=4$, there remain the characters denoted $D_{4}, r$ and $\phi_{80,7}$ with $\ell=5$. For $d=6$, we are left with $\phi_{6,25}$. When $G={ }^{2} E_{6}(q), E_{7}(q)$ or $E_{8}(q)$, a completely similar argument leads to the other three candidates in Table 4 . 
Remark 6.10. Using decomposition numbers of suitable Hecke algebras one can see that the unipotent characters $\phi_{80,7}$ for $E_{6}(q)$ and $\phi_{16,5}$ for ${ }^{2} E_{6}(q)$ are reducible modulo primes $\ell$ with $d_{\ell}(q)=4$, so they are not endotrivial. On the other hand, the unipotent character $F_{4}^{I I}[1]$ is endotrivial modulo $\ell=5$ at least for $q=2$ (see Remark 7.2). We do not see how to decide endotriviality for the other cases in Table 4.

We are now ready to complete the proof of Theorem [1.2(d) of the introduction:

Theorem 6.11. Let $G$ be a quasi-simple exceptional group of Lie type in characteristic $p$, and $\ell \neq p$ a prime such that the Sylow $\ell$-subgroups of $G$ have rank at least 3 . Then $G$ does not have faithful simple endotrivial $k G$-modules, where $k$ is a field of characteristic $\ell$.

Proof. By Theorem 6.8 we may assume that $G$ is of rank at least 4 . If $G$ is an exceptional covering group $2 . F_{4}(2)$ or $2 .{ }^{2} E_{6}(2)$ then the condition on $\ell$ forces $\ell=3$. Here the claim follows from the known ordinary character tables. Thus we have that $G$ is a central factor group of a finite reductive group of simply connected type. Let $d=d_{\ell}(q)$. Then the cases to consider are: $d=1,2$ for $F_{4}(q), d=1,2,3$ for $E_{6}(q), d=1,2,6$ for ${ }^{2} E_{6}(q), d=1,2,3,6$ for $E_{7}(q)$ and $d=1,2,3,4,6$ for $E_{8}(q)$.

Let $V$ be a faithful simple endotrivial $k G$-module, the $\ell$-modular reduction of a $\mathbb{C} G$ module with character $\chi$. Since all candidates in Proposition 6.9 occur for $\ell$-rank 2 , we know that $\chi$ is not unipotent, so $\chi$ lies in some Lusztig series $\mathcal{E}(G, s)$ with $s \neq 1$. Furthermore, $s$ must lie in the centralizer of a Sylow $d$-torus of $G^{*}$ by Proposition 6.3. In all cases considered here, $d$ is a Springer regular number for the Weyl group of $G$ (see e.g. [6, p. 260]), so the centralizer of a Sylow $d$-torus of $G^{*}$ is a maximal torus $T^{*}$ of $G^{*}$. Moreover, a Sylow $d$-torus of $G^{*}$ is already a maximal torus, whence equals $T^{*}$, unless $G=E_{6}(q)$ and $d=2, G={ }^{2} E_{6}(q)$ and $d=1$, or $G=E_{7}(q)$ and $d=3,6$. In Table 5 in each relevant case we list two or three maximal tori of $G^{*}$ (in Carter's notation, see also [17]). We have omitted ${ }^{2} E_{6}(q)$ since the relevant tori in that group are obtained from those in $E_{6}(q)$ by formally replacing $q$ by $-q$ (see $[6, \S 3 \mathrm{~B}]$ ).

TABLE 5. Large $\ell$-rank in exceptional groups

\begin{tabular}{|ccl||rll|}
\hline$G$ & $d$ & tori & $G$ & $d$ & tori \\
\hline$F_{4}$ & 1 & $A_{2}+\tilde{A}_{1}, \tilde{A}_{2}+A_{1}, A_{3}$ & $E_{6}$ & 1 & $D_{5}, A_{4}+A_{1}$ \\
& 2 & $B_{3}, C_{3}, A_{3}$ & & 2 & $D_{5}, A_{4}+A_{1}$ \\
$E_{8}$ & 1 & $E_{7}, A_{7}^{\prime \prime}$ & & 3 & $A_{2}+2 A_{1}, E_{6}$ \\
& 2 & $A_{8}, A_{7}^{\prime \prime}$ & $E_{7}$ & 1 & $E_{6}\left(a_{1}\right), A_{6}$ \\
& 3 & $A_{8}, D_{7}$ & & 2 & $E_{7}, E_{7}\left(a_{1}\right)$ \\
& 4 & $D_{7}\left(a_{1}\right), A_{7}, A_{7}^{\prime \prime}$ & & 3 & $A_{4}+A_{2}, E_{6}$ \\
& 6 & $E_{8}\left(a_{4}\right), D_{7}$ & & 6 & $E_{7}\left(a_{2}\right), E_{7}\left(a_{3}\right)$ \\
\hline
\end{tabular}

Now in all cases, except when $G=E_{8}(q)$ and $d=4$, the only element $s \in T^{*}$ such that $C_{G^{*}}(s)$ contains conjugates of the two (or three) tori listed in the table, is $s=1$. This is easily checked by using that maximal tori are parametrized by (possibly twisted) conjugacy classes in the Weyl group; thus one just has to verify that the Weyl coset of any centralizer $C_{G^{*}}(s)$ does not contain representatives from all two or three conjugacy 
classes. Since all listed tori do contain regular elements, Proposition 6.4 implies that $\chi \in \mathcal{E}(G, s)$ cannot be endotrivial when $s \neq 1$ and $(G, d) \neq\left(E_{8}(q), 4\right)$.

When $G=E_{8}(q)$ and $d=4$, there is an isolated element $s \in T^{*}$ of order 2 whose centralizer $D_{8}(q)$ contains all listed maximal tori. It remains to show that $\mathcal{E}(G, s)$ for this element $s$ does not contain characters of endotrivial modules. As $d=4$ we have $\ell \geq 5$. Now the approach given for unipotent characters in the proof of Proposition 6.9 using congruences and Harish-Chandra restriction rules out all characters in this series.

\section{Covering Groups of SPORADIC Simple Groups}

Theorem 7.1. Let $G$ be a quasi-simple group such that $G / Z(G)$ is sporadic simple. Let $V$ be a faithful simple endotrivial $k G$-module, where $k$ is algebraically closed of characteristic $p$, with $p$ dividing $|G|$. Let $P$ be a Sylow p-subgroup of $G$. Then one of the following holds:

(1) $|P|=p$ and $V$ lies in a $p$-block $\mathbf{B}$ of $k G$ as indicated in Table 7 ; or

(2) $(G, P, \operatorname{dim} V)$ are as in Table 6 .

Conversely, all modules listed in Table [6] are endotrivial except possibly for those (of dimension at least 5824) marked by a "?" in the last column.

Table 7 also gives the number se $(\mathbf{B})$ of simple endotrivial modules in the block $\mathbf{B}$, except for one block of $2 . B$ in characteristic 47 and five blocks of $M$ in various characteristics, where the Brauer trees are not known completely. The numbering of the blocks is as given by Hiß and Lux in [24].

Proof. First assume $p^{2}$ divides $|G|$. Let $\chi$ be the ordinary irreducible character of $G$ belonging to the lift of $V$ from Theorem 1.3. Using Lemma 2.1 and Corollary 2.3 and the known ordinary character tables of the quasi-simple sporadic groups (see [12]) we obtain the list of candidates for $\chi$.

Using the character tables given in [30, 43] we may discard the characters whose reduction modulo $p$ is not irreducible. Thus Table 6 lists those characters whose restriction modulo $p$ is irreducible, or, in the case of $J_{4}, F i_{24}^{\prime}, B$ and $M$, those for which the question of irreducibility modulo $p$ is still open (these cases are indicated by a question mark in the corresponding line). The sheer size of these modules makes it impossible to do any direct computations.

The modules for $M_{11}, M_{22}, 2 . M_{22}, M_{23}$ with $p=3$ are indeed endotrivial by [42, §2.3]. For the cases

$$
\begin{aligned}
(G, p, \chi(1)) \in\{ & \left(2 . M_{22}, 3,10\right),(3 . M c L, 5,126),(2 . R u, 3,28),(3 . O N, 7,342), \\
& \left.\left({ }^{2} F_{4}(2)^{\prime}, 3,26\right),\left({ }^{2} F_{4}(2)^{\prime}, 5,26\right)\right\}
\end{aligned}
$$

it can be seen from the character table that the tensor product $\chi \otimes \chi^{*}$ has one trivial constituent and one constituent of defect zero (see also [33, Table 7.1]), so clearly the corresponding $k G$-module $V$ is endotrivial. For the following configurations the restriction of the corresponding ordinary character to a subgroup $H$ containing a Sylow $p$-subgroup of $G$ has a unique trivial constituent, and all other constituents are of defect zero:

$$
\begin{aligned}
(G, p, \chi(1), H) \in & \left\{\left(M_{23}, 3,253, M_{22}\right),\left(H e, 5,51, \mathrm{~S}_{4}(4) .2\right),\left(R u, 3,406,{ }^{2} F_{4}(2)^{\prime}\right),\right. \\
& \left(S u z, 5,1001, G_{2}(4)\right),\left(F i_{22}, 5,1001, \mathrm{O}_{8}^{+}(2) \cdot \mathfrak{S}_{3}\right),\left(3 . F i_{22}, 5,351, \mathrm{O}_{8}^{+}(2)\right), \\
& \left.\left(F i_{23}, 5,111826, \mathrm{O}_{8}^{+}(3) . \mathfrak{S}_{3}\right)\right\}
\end{aligned}
$$


TABLE 6. Candidate characters in sporadic groups

\begin{tabular}{|c|l|l||c|l|l|l|}
\hline$G$ & $P$ & $\chi(1)$ & $G$ & $P$ & $\chi(1)$ & \\
\hline$M_{11}$ & $3^{2}$ & $10,10,10$ & $F i_{22}$ & $5^{2}$ & 1001 & \\
$M_{22}$ & $3^{2}$ & 55 & $2 . F i_{22}$ & $5^{2}$ & $5824(4 \times)$ & $?$ \\
$2 . M_{22}$ & $3^{2}$ & $10,10,154,154$ & $3 . F i_{22}$ & $5^{2}$ & $351,351,12474(4 \times)$ & $?$ \\
$M_{23}$ & $3^{2}$ & 253 & $6 . F i_{22}$ & $5^{2}$ & $61776(4 \times)$ & $?$ \\
$H S$ & $3^{2}$ & $154,154,154$ & $T h$ & $7^{2}$ & 27000,27000 & $?$ \\
$3 . M c L$ & $5_{+}^{1+2}$ & $126,126,126,126$ & $F i_{23}$ & $5^{2}$ & 111826 & \\
$H e$ & $5^{2}$ & 51,51 & $J_{4}$ & $11_{+}^{1+2}$ & $887778,887778,394765284$ & $?$ \\
$R u$ & $3_{+}^{1+2}$ & 406 & $F i_{24}^{\prime}$ & $5^{2}$ & 74887473024 & $?$ \\
$2 . R u$ & $3_{+}^{1+2}$ & 28,28 & $B$ & $7^{2}$ & 9287037474,775438738408125 & $?$ \\
$S u z$ & $5^{2}$ & 1001 & $M$ & $11^{2}$ & 7226910362631220625000 & $?$ \\
$3 . O N$ & $7_{+}^{1+2}$ & $342,342,342,342$ & ${ }^{2} F_{4}(2)^{\prime}$ & $3_{+}^{1+2}$ & 26,26 & \\
& & ${ }^{2} F_{4}(2)^{\prime}$ & $5^{2}$ & $26,26,351,351$ & \\
\hline
\end{tabular}

so $\chi$ is the character of an endotrivial module by Lemma 2.2. Computations with Magma [32] show that the modules $(G, p, \chi(1)) \in\left\{(H S, 3,154),\left({ }^{2} F_{4}(2)^{\prime}, 5,351\right)\right\}$ are endotrivial.

Finally, we consider the cases with cyclic Sylow $p$-subgroup. The results are collected in Table 7, whose entries are obtained as follows. Let $H:=N_{G}(Z)$ as in Section 3 , $|X(H)|=|H /[H, H]|_{p^{\prime}}$ and let $e$ denote the inertial index of the principal block. Then $|X(H)|$ and $|X(H)| / e$ are the bounds for the number of simple endotrivial $k G$-modules given by Corollary 3.3. We then determine the simple endotrivial $k G$-modules using the descriptions of the Brauer trees in [24, resp. 38] for $M$ in characteristic 29, as well as Lemma 3.2, Lemma 3.1, Theorem 3.7, Lemma 2.1 and Lemma 2.3.

Remark 7.2. If $V$ is the reduction modulo $p$ of a $\mathbb{C} G$-module with character $\chi$ such that

$$
\begin{aligned}
(G, p, \chi(1), H) \in & \left\{\left(M_{23}, 3,253, M_{22}\right),\left(H e, 5,51, \mathrm{~S}_{4}(4) .2\right),\left(R u, 3,406,{ }^{2} F_{4}(2)^{\prime}\right),\right. \\
& \left(S u z, 5,1001, G_{2}(4)\right),\left(F i_{22}, 5,1001, \mathrm{O}_{8}^{+}(2) \cdot \mathfrak{S}_{3}\right),\left(3 . F i_{22}, 5,351, \mathrm{O}_{8}^{+}(2)\right), \\
& \left.\left(F i_{23}, 5,111826, \mathrm{O}_{8}^{+}(3) . \mathfrak{S}_{3}\right)\right\}
\end{aligned}
$$

as in the proof of Theorem 7.1, then $V$ is not only endotrivial, but also a trivial source $k G$-module because $\left.V\right|_{H} \cong k \oplus$ (projective). Therefore the class of $V$ in the group $T(G)$ of endotrivial modules is a torsion element. Another such example is given by the unipotent character $F_{4}^{I I}[1]$ of $F_{4}(2)$ of degree 1326 from Proposition 6.9 (with the subgroup $H=$ $\left.\mathrm{O}_{8}^{+}(2) .3 .2\right)$.

Thus we have obtained a list of non-trivial torsion elements of $T(G)$ unknown in the literature so far. This is of particular interest because a main problem remaining in the classification of endotrivial modules is the determination of the torsion subgroup of $T(G)$.

Remark 7.3. Endotriviality is in general not preserved by Morita equivalences. Let us point out the following example: the Janko group $J_{1}$ has four 3-blocks with isomorphic Brauer trees, that is, isomorphic as pointed graphs equipped with a planar embedding. Hence the four blocks are Morita equivalent (see [24, pp. 69-70]). However, Table 7 shows that only two of these blocks contain simple endotrivial modules. 


\section{REFERENCES}

[1] J. L. Alperin, Lifting endo-trivial modules. J. Group Theory 4 (2001), 1-2.

[2] D. J. Benson, Representations and Cohomology I. Cambridge Studies in Advanced Mathematics, 30. Cambridge University Press, Cambridge, 1998.

[3] C. Bessenrodt, Modular representation theory for blocks with cyclic defect groups via the Auslander-Reiten quiver. J. Algebra 140 (1991), 247-262.

[4] C. Bessenrodt, Endotrivial modules and the Auslander-Reiten quiver. Pp. 317-326 in: Representation Theory of Finite Groups and Finite-Dimensional Algebras (Bielefeld, 1991), Progr. Math., 95, Birkhäuser, Basel, 1991.

[5] C. Bonnafé, Representations of $\mathrm{SL}_{2}\left(\mathbb{F}_{q}\right)$. Algebra and Applications, 13. Springer-Verlag London Ltd., London, 2011.

[6] M. Broué, G. Malle, Théorèmes de Sylow génériques pour les groupes réductifs sur les corps finis. Math. Ann. 292 (1992), 241-262.

[7] J. Carlson, Endotrivial modules. Pp. 99-111 in: Recent Developments in Lie Algebras, Groups and Representation Theory. Proc. Sympos. Pure Math., Providence, RI, 2012.

[8] J. Carlson, D, Hemmer, N. Mazza, The group of endotrivial modules for the symmetric and alternating groups. Proc. Edinb. Math. Soc. 53 (2010), 83-95.

[9] J. Carlson, N. Mazza, D. Nakano, Endotrivial modules for finite groups of Lie type. J. Reine Angew. Math. 595 (2006), 93-119.

[10] J. Carlson, N. Mazza, D. Nakano, Endotrivial modules for the symmetric and alternating groups. Proc. Edinb. Math. Soc. (2). 52 (2009), 45-66.

[11] R. CARTER, Finite Groups of Lie type: Conjugacy Classes and Complex Characters. Wiley, Chichester, 1985.

[12] J.H. Conway, R.T. Curtis, S.P. Norton, R.A. Parker, R.A. Wilson, Atlas of Finite Groups. Clarendon Press, Oxford, 1985.

[13] D. A. Craven, Algebraic Modules for Finite Groups. D. Phil. thesis, Mathematical Institute, University of Oxford, 2007.

[14] E. C. DADE, Endo-permutation modules over p-groups, II. Ann. of Math. (2) 108 (1978), 317-346.

[15] K. Erdmann, Young modules for symmetric groups. J. Aust. Math. Soc. 71 (2001), 201-210.

[16] W. FEIT, The Representation Theory of Finite Groups. North-Holland Mathematical Library, 25. North-Holland Publishing Co., Amsterdam, 1982.

[17] M. Geck, G. Hiss, F. Lübeck, G. Malle, G. Pfeiffer, CHEVIE - A system for computing and processing generic character tables for finite groups of Lie type, Weyl groups and Hecke algebras. Appl. Algebra Engrg. Comm. Comput. 7 (1996), 175-210.

[18] R. M. Guralnick, G. Malle, Products of conjugacy classes and fixed point spaces. J. Amer. Math. Soc. 25 (2012), 77-121.

[19] D. Hemmer, Irreducible Specht modules are signed Young modules. J. Algebra 305 (2006), 433441.

[20] A. Henke, On p-Kostka numbers and Young modules. European J. Combin. 26 (2005), 923-942.

[21] F. Himstedt, On the decomposition numbers of the Ree groups ${ }^{2} F_{4}\left(q^{2}\right)$ in non-defining characteristic. J. Algebra 325 (2011), 364-403.

[22] G. Hiss, Zerlegungszahlen endlicher Gruppen vom Lie-Typ in nicht-definierender Charakteristik. Habilitationsschrift, RWTH Aachen, 1990.

[23] G. Hiss, Hermitian function fields, classical unitals, and representations of 3-dimensional unitary groups. Indag. Math. (N.S.) 15 (2004), 223-243.

[24] G. Hiss, K. Lux, Brauer Trees of Sporadic Groups. Oxford Science Publications. The Clarendon Press, Oxford University Press, New York, 1989.

[25] P. N. Hoffman, J. F. Humphreys, Projective Representations of the Symmetric Groups. QFunctions and Shifted Tableaux. Oxford Science Publications. The Clarendon Press, Oxford University Press, New York, 1992.

[26] J. E. Humphreys, Introduction to Lie Algebras and Representation Theory. Second printing, revised. Springer-Verlag, New York-Berlin, 1978. 
TABLE 7. Blocks of sporadic groups with cyclic defect containing simple endotrivial modules

\begin{tabular}{|r|r|r|c|c|c|}
\hline$G$ & $p$ & $|X(H)|$ & $|X(H)| / e$ & block B & se(B) \\
\hline \hline${ }^{2} F_{4}(2)^{\prime}$ & 13 & 6 & 1 & 1 & 4 \\
\hline \hline$M_{11}$ & 5 & 4 & 1 & 1 & 4 \\
\cline { 2 - 6 }$M_{11}$ & 11 & 5 & 1 & 1 & 3 \\
\hline \hline$M_{12}$ & 5 & 8 & 2 & 1 & 2 \\
$M_{12}$ & 5 & & & 2 & 4 \\
\cline { 2 - 6 }$M_{12}$ & 11 & 5 & 1 & 1 & 1 \\
$2 . M_{12}$ & 11 & 10 & 2 & 2 & 4 \\
\hline \hline$J_{1}$ & 3 & 4 & 2 & 1,4 & 2 \\
\cline { 2 - 6 }$J_{1}$ & 5 & 4 & 2 & 1 & 2 \\
$J_{1}$ & 5 & & & 3 & 1 \\
\cline { 2 - 6 }$J_{1}$ & 7 & 6 & 1 & 1 & 2 \\
\cline { 2 - 6 }$J_{1}$ & 11 & 10 & 1 & 1 & 2 \\
\cline { 2 - 6 }$J_{1}$ & 19 & 6 & 1 & 1 & 2 \\
\hline \hline$M_{22}$ & 5 & 4 & 1 & 1 & 2 \\
$2 . M_{22}$ & 5 & 8 & 2 & 2 & 2 \\
$3 . M_{22}$ & 5 & 12 & 3 & 3,4 & 2 \\
$4 . M_{22}$ & 5 & 16 & 4 & 5,6 & 2 \\
$6 . M_{22}$ & 5 & 24 & 6 & 7,8 & 4 \\
$12 . M_{22}$ & 5 & 48 & 12 & $9,10,11,12$ & 3 \\
\cline { 2 - 6 }$M_{22}$ & 7 & 3 & 1 & 1 & 1 \\
$2 . M_{22}$ & 7 & 6 & 2 & 2 & 1 \\
$3 . M_{22}$ & 7 & 9 & 3 & 3,4 & 1 \\
$4 . M_{22}$ & 7 & 12 & 4 & 5,6 & 1 \\
$6 . M_{22}$ & 7 & 18 & 6 & 7,8 & 1 \\
$12 . M_{22}$ & 7 & 36 & 12 & $9,10,11,12$ & 2 \\
\cline { 2 - 6 }$M_{22}$ & 11 & 5 & 1 & 1 & 3 \\
$2 . M_{22}$ & 11 & 10 & 2 & 2 & 3 \\
$3 . M_{22}$ & 11 & 15 & 3 & 3,4 & 4 \\
$4 . M_{22}$ & 11 & 20 & 4 & 5,6 & 4 \\
$6 . M_{22}$ & 11 & 30 & 6 & 7,8 & 2 \\
$12 . M_{22}$ & 11 & 60 & 12 & 9,10 & 2 \\
$12 . M_{22}$ & 11 & & & 11,12 & 4 \\
\hline \hline$J_{2}$ & 7 & 6 & 1 & 1 & 2 \\
$2 . J_{2}$ & 7 & 12 & 2 & 2 & 4 \\
\hline \hline$M_{23}$ & 5 & 4 & 1 & 1 & 4 \\
\cline { 2 - 6 }$M_{23}$ & 7 & 8 & 2 & 1 & 1 \\
\cline { 2 - 6 }$M_{23}$ & 11 & 5 & 1 & & 5 \\
\cline { 2 - 6 }$M_{23}$ & 11 & 1 & & \\
\hline
\end{tabular}




\begin{tabular}{|c|c|c|c|c|c|}
\hline$G$ & $p$ & $|X(H)|$ & $|X(H)| / e$ & block B & $\mathrm{se}(\mathbf{B})$ \\
\hline$\overline{H S S}$ & $\overline{77}$ & 6 & 1 & 1 & 2 \\
\hline 2.HS & 7 & 12 & 2 & 2 & 2 \\
\hline$H S$ & 11 & 5 & 1 & 1 & 1 \\
\hline 2.HS & 11 & 10 & 2 & 2 & 2 \\
\hline$J_{3}$ & $\overline{5}$ & 4 & 2 & 1,3 & 1 \\
\hline $3 . J_{3}$ & 5 & 12 & 6 & $4,5,6,7$ & 1 \\
\hline$J_{3}$ & 17 & 8 & 1 & 1 & 2 \\
\hline $3 . J_{3}$ & 17 & 24 & 3 & 2,3 & 6 \\
\hline$J_{3}$ & 19 & 9 & 1 & 1 & 1 \\
\hline $3 . J_{3}$ & 19 & 27 & 3 & 2,3 & 6 \\
\hline$M_{24}$ & 5 & 4 & 1 & 1 & 2 \\
\hline$M_{24}$ & 7 & 6 & 2 & 1,3 & 1 \\
\hline$M_{24}$ & 11 & 10 & 1 & 1 & 6 \\
\hline$M_{24}$ & 23 & 11 & 1 & 1 & 7 \\
\hline $\bar{M} M c L$ & 7 & $\overline{66}$ & "2 & 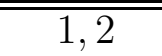 & 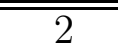 \\
\hline 3.McL & 7 & 18 & 6 & 3,4 & 1 \\
\hline 3.McL & 7 & & & 5,6 & 2 \\
\hline$M c L$ & 11 & 5 & 1 & 1 & 1 \\
\hline 3.McL & 11 & 15 & 3 & 2,3 & 1 \\
\hline $\mathrm{He}$ & 17 & 8 & 1 & 1 & 1 \\
\hline$\overline{\bar{R} R u}$ & $\overline{77}$ & 6 & 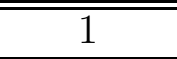 & $\overline{1}$ & 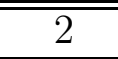 \\
\hline$R u$ & 13 & 12 & 1 & 1 & 4 \\
\hline$R u$ & 29 & 14 & 1 & 1 & 4 \\
\hline $2 . R u$ & 29 & 28 & 2 & 3 & 9 \\
\hline$\overline{S \text { Suz }}$ & $\overline{7}$ & $\overline{66}$ & $\overline{1}$ & 1 & 2 \\
\hline 3.Suz & 7 & & & 8,9 & 2 \\
\hline Suz & 11 & 10 & 1 & 1 & 2 \\
\hline 2.Suz & 11 & 20 & 2 & 2 & 4 \\
\hline 3.Suz & 11 & 30 & 3 & 3,4 & 6 \\
\hline 6.Suz & 11 & 60 & 6 & 5,6 & 6 \\
\hline Suz & 13 & 6 & 1 & 1 & 1 \\
\hline 2.Suz & 13 & 12 & 2 & 2 & 1 \\
\hline 3.Suz & 13 & 18 & 3 & 3,4 & 1 \\
\hline 6.Suz & 13 & 36 & 6 & 5,6 & 3 \\
\hline 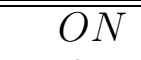 & $\bar{~} 5$ & 8 & 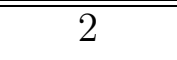 & 1 & 2 \\
\hline$O N$ & 5 & & & 2 & 4 \\
\hline$O N$ & 11 & 10 & 1 & 1 & 2 \\
\hline $3 . O N$ & 11 & 30 & 3 & 2,3 & 4 \\
\hline$O N$ & 19 & 6 & 1 & 1 & 2 \\
\hline $3 . O N$ & 19 & 18 & 3 & 2,3 & 3 \\
\hline$O N$ & 31 & 15 & 1 & 1 & 1 \\
\hline $3 . O N$ & 31 & 45 & 3 & 2,3 & 8 \\
\hline
\end{tabular}




\begin{tabular}{|c|c|c|c|c|c|}
\hline$G$ & $p$ & $|X(H)|$ & $|X(H)| / e$ & block B & $\mathrm{se}(\mathbf{B})$ \\
\hline $\mathrm{CO}_{3}$ & $\overline{77}$ & 12 & 2 & $\overline{11}$ & 4 \\
\hline $\mathrm{CO}_{3}$ & 7 & & & 3 & 2 \\
\hline $\mathrm{Co}_{3}$ & 11 & 10 & 2 & 1,2 & 1 \\
\hline $\mathrm{Co}_{3}$ & 23 & 11 & 1 & 1 & 5 \\
\hline$\overline{\mathrm{CO}_{2}}$ & 7 & $\overline{12}$ & 2 & 1,3 & 2 \\
\hline $\mathrm{CO}_{2}$ & 11 & 10 & 1 & 1 & 4 \\
\hline $\mathrm{CO}_{2}$ & 23 & 11 & 1 & 1 & 5 \\
\hline$F i_{22}$ & 7 & $\overline{12}$ & 2 & 1,3 & 2 \\
\hline 2. $F i_{22}$ & 7 & 24 & 4 & 5,6 & 2 \\
\hline 3. $F i_{22}$ & 7 & 36 & 6 & $7,8,9,10$ & 2 \\
\hline 6. $F i_{22}$ & 7 & 72 & 12 & $13,14,15,16$ & 2 \\
\hline$F i_{22}$ & 11 & 10 & 2 & 1,2 & 1 \\
\hline 2.Fi $i_{22}$ & 11 & 20 & 4 & 3,4 & 1 \\
\hline 3. $F i_{22}$ & 11 & 30 & 6 & $5,6,7,8$ & 2 \\
\hline $6 . F i_{22}$ & 11 & 60 & 12 & $9,10,11,12$ & 2 \\
\hline$F i_{22}$ & 13 & 6 & 1 & 1 & 1 \\
\hline 2.Fi $i_{22}$ & 13 & 12 & 2 & 2 & 4 \\
\hline 3. $F i_{22}$ & 13 & 18 & 3 & 3,4 & 1 \\
\hline $6 . F i_{22}$ & 13 & 36 & 6 & 5,6 & 2 \\
\hline "HN & $\overline{77}$ & $\overline{6}$ & $\overline{1}$ & 1 & $\overline{2}$ \\
\hline$H N$ & 11 & 20 & 2 & 1 & 2 \\
\hline$H N$ & 11 & & & 2 & 4 \\
\hline$H N$ & 19 & 9 & 1 & 1 & 1 \\
\hline$\overline{L L y}$ & $\overline{\overline{7}}$ & $\overline{\bar{c}}$ & $\overline{1} 1$ & $\overline{c 1}$ & 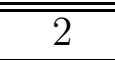 \\
\hline Ly & 11 & 10 & 2 & 1,2 & 1 \\
\hline Ly & 31 & 6 & 1 & 1 & 4 \\
\hline Ly & 37 & 18 & 1 & 1 & 6 \\
\hline Ly & 67 & 22 & 1 & 1 & 6 \\
\hline$\overline{\overline{T h}}$ & 13 & 12 & $\overline{\overline{1}}$ & 1 & 8 \\
\hline$T h$ & 19 & 18 & 1 & 1 & 10 \\
\hline$T h$ & 31 & 15 & 1 & 1 & 11 \\
\hline$\overline{\overline{F i} i_{23}}$ & 7 & $\overline{\overline{12}}$ & 2 & $\overline{1,4}$ & 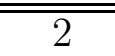 \\
\hline$F i_{23}$ & 11 & 20 & 2 & 1 & 2 \\
\hline$F i_{23}$ & 11 & & & 3 & 4 \\
\hline$F i_{23}$ & 13 & 12 & 2 & 1,3 & 2 \\
\hline$F i_{23}$ & 17 & 16 & 1 & 1 & 4 \\
\hline$F i_{23}$ & 23 & 11 & 1 & 1 & 1 \\
\hline$\overline{C C_{o_{1}}}$ & 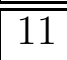 & $\overline{20}$ & 2 & $\overline{\overline{1}}$ & 2 \\
\hline $\mathrm{Co}_{1}$ & 11 & & & 2 & 4 \\
\hline$C_{o_{1}}$ & 13 & 12 & 1 & 1 & 4 \\
\hline$C_{o_{1}}$ & 23 & 11 & 1 & 1 & 1 \\
\hline 2.Co $o_{1}$ & 23 & 22 & 2 & 2 & 5 \\
\hline
\end{tabular}




\begin{tabular}{|c|c|c|c|c|c|}
\hline$G$ & $p$ & $|X(H)|$ & $|X(H)| / e$ & block B & $\mathrm{se}(\mathbf{B})$ \\
\hline 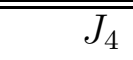 & 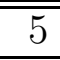 & $\begin{array}{r}4 \\
\end{array}$ & 1 & 1 & $\overline{2}$ \\
\hline$J_{4}$ & 7 & 6 & 2 & 1 & 1 \\
\hline$J_{4}$ & 7 & & & 2 & 1 \\
\hline$J_{4}$ & 23 & 22 & 1 & 1 & 10 \\
\hline$J_{4}$ & 29 & 28 & 1 & 1 & 10 \\
\hline$J_{4}$ & 31 & 10 & 1 & 1 & 2 \\
\hline$J_{4}$ & 37 & 12 & 1 & 1 & 6 \\
\hline$J_{4}$ & 43 & 14 & 1 & 1 & 8 \\
\hline$\overline{F F i_{24}^{\prime}}$ & $\overline{111}$ & $\overline{10}$ & 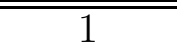 & 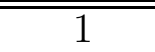 & 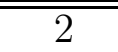 \\
\hline $3 . F_{24}^{\prime}$ & 11 & 30 & 3 & 8,9 & 4 \\
\hline$F i_{24}^{\prime}$ & 13 & 24 & 2 & 1,4 & 4 \\
\hline$F i_{24}^{\prime}$ & 17 & 16 & 1 & 1 & 2 \\
\hline 3. $F i_{24}^{\prime}$ & 17 & 48 & 3 & 2,3 & 6 \\
\hline$F i_{24}^{\prime}$ & 23 & 11 & 1 & 1 & 1 \\
\hline 3. $F i_{24}^{\prime}$ & 23 & 33 & 3 & 2,3 & 5 \\
\hline$F i_{24}^{\prime}$ & 29 & 14 & 1 & 1 & 3 \\
\hline 3. $F i_{24}^{\prime}$ & 29 & 42 & 3 & 2,3 & 5 \\
\hline$B$ & 11 & 20 & 2 & 1,7 & 2 \\
\hline$B$ & 13 & 24 & 2 & 1,5 & 2 \\
\hline$B$ & 17 & 32 & 2 & 1 & 6 \\
\hline$B$ & 17 & & & 3 & 4 \\
\hline$B$ & 19 & 36 & 2 & 1 & 4 \\
\hline$B$ & 19 & & & 2 & 6 \\
\hline$B$ & 23 & 22 & 2 & 1,2 & 1 \\
\hline $2 . B$ & 23 & 44 & 4 & 3 & 5 \\
\hline $2 . B$ & 23 & & & 4 & 7 \\
\hline$B$ & 31 & 15 & 1 & 1 & 1 \\
\hline $2 . B$ & 31 & 30 & 2 & 2 & 9 \\
\hline$B$ & 47 & 23 & 1 & 1 & 5 \\
\hline 2.B & 47 & 46 & 2 & 2 & $\geq 3$ \\
\hline$\overline{\bar{M}}$ & $\bar{~} 17$ & 16 & 1 & 1 & $\bar{~} 6$ \\
\hline$M$ & 19 & 18 & 1 & 1 & 6 \\
\hline$M$ & 23 & 22 & 2 & 1 & 5 \\
\hline$M$ & 23 & & & 5 & 1 \\
\hline$M$ & 29 & 28 & 1 & 1 & $\geq 10$ \\
\hline$M$ & 31 & 30 & 2 & 1 & 3 \\
\hline$M$ & 31 & & & 3 & 5 \\
\hline$M$ & 41 & 40 & 1 & 1 & $\geq 8$ \\
\hline$M$ & 47 & 46 & 2 & 1 & $\geq 3$ \\
\hline$M$ & 47 & & & 2 & 9 \\
\hline$M$ & 59 & 29 & 1 & 1 & $\geq 15$ \\
\hline$M$ & 71 & 35 & 1 & 1 & $\geq 7$ \\
\hline
\end{tabular}


[27] B. Huppert, N. Blackburn, Finite groups. II. Grundlehren der Mathematischen Wissenschaften 242. Springer-Verlag, Berlin-New York, 1982.

[28] G. D. James, On a conjecture of Carter concerning irreducible Specht modules. Math. Proc. Cambridge Philos. Soc. 83 (1978), 11-17.

[29] G. D. James, A. Kerber, The Representation Theory of the Symmetric Group. Encyclopedia of Mathematics and its Applications, 16. Addison-Wesley Publishing Co., Reading, Mass., 1981.

[30] C. Jansen, K. Lux, R. Parker, R. Wilson, An Atlas of Brauer Characters. Oxford Science Publications. The Clarendon Press, Oxford University Press, New York, 1995.

[31] P. Landrock, G. Michler, Principal 2-blocks of the simple groups of Ree type. Trans. Amer. Math. Soc. 260 (1980), 83-111.

[32] MAGMA V2.12-14, The computational Algebra Group, School of Mathematics and Statistics, University of Sydney. http://magma.maths .usyd.edu.au/magma/.

[33] G. MAlle, Almost irreducible tensor squares. Comm. Algebra 27 (1999), 1033-1051.

[34] G. MALle, Height 0 characters of finite groups of Lie type. Represent. Theory 11 (2007), 192-220.

[35] G. Malle, D. Testerman, Linear Algebraic Groups and Finite Groups of Lie Type. Cambridge Studies in Advanced Mathematics, 133. Cambridge University Press, Cambridge, 2011.

[36] N. Mazza, J. Thévenaz, Endotrivial modules in the cyclic case. Arch. Math. (Basel) 89 (2007), 497-503.

[37] J. MülLER, Brauer trees for the Schur cover of the symmetric group. J. Algebra 266 (2003), $427-445$.

[38] M. NAenrig, Die Brauerbäume des Monsters $M$ in Charakteristik 29. Diplomarbeit, Lehrstuhl D für Mathematik, RWTH Aachen, 2002.

[39] G. Navarro, G. Robinson, On endo-trivial modules for p-solvable groups. Math. Z. 270 (2012), 983-987.

[40] M. H. PeEL, Hook representations of the symmetric groups. Glasgow Math. J. 12 (1971), 136-149.

[41] G. Robinson, On simple endotrivial modules. Bull. Lond. Math. Soc. 43 (2011), 712-716.

[42] E. Schulte, Simple Endotrivial Modules for Finite Simple Groups. Diplomarbeit, TU Kaiserslautern, 2012.

[43] The GAP Group, GAP - Groups, Algorithms, and Programming, Version 4.4; 2004, http: //www.gap-system.org.

[44] J. ThévenAz, G-Algebras and Modular Representation Theory. Oxford Science Publications. The Clarendon Press, Oxford University Press, New York, 1995.

[45] J. Thévenaz, Endo-permutation modules, a guided tour. Pp. 115-147 in: Group Representation Theory. EPFL Press, Lausanne, 2007.

[46] P. H. TieP, A. E. Zalesski, Real conjugacy classes in algebraic groups and finite groups of Lie type. J. Group Theory 8 (2005), 291-315.

[47] J.-M. Urfer, Groupe de Dade et Modules Endo-Triviaux. Travail de diplôme, Université de Lausanne, 2002.

FB Mathematik, TU Kaiserslautern, Postfach 3049, 67653 Kaiserslautern, Germany.

E-mail address: lassueur@mathematik.uni-kl.de

E-mail address: malle@mathematik.uni-kl.de

E-mail address: eschulte@mathematik.uni-kl.de 